03

\title{
Излучение в дальней зоне элементарного излучателя, расположенного на границе плоскослоистой структуры
}

\author{
(C) А.Б. Петрин \\ Объединенный институт высоких температур РАН, \\ 125412 Москва, Россия \\ e-mail: a_petrin@mail.ru \\ Поступила в редакцию 28.08.2020 г. \\ В окончательной редакции 28.08.2020 г. \\ Принята к публикации 04.09.2020 г.
}

\begin{abstract}
Рассмотрена строгая теория нахождения излучения в дальней зоне элементарного диполя, расположенного на границе или внутри плоскослоистой структуры. Подробно рассмотрен частный случай излучения элементарного диполя, расположенного на свободной границе одной пленки, предложен эффективный метод нахождения поля излучения в дальней зоне в окружающих полупространствах. Получены диаграммы направленности точечных излучателей (молекул, наноструктур), расположенных на свободной поверхности металлической пленки в схеме Кречмана и имеющих индуцированный дипольный момент, направленный параллельно или перпендикулярно поверхности пленки.
\end{abstract}

Ключевые слова: нанофокусировка, поверхностные плазмоны, оптические сенсоры.

DOI: $10.21883 /$ OS.2020.12.50325.226-20

\section{Введение}

В настоящее время сохраняется значительный интерес к физическим явлениям, которые сопровождают возбуждение поверхностной плазмонной волны на поверхности металлической пленки по схеме Кречмана [1] (рис. 1). Поверхностная плазмонная волна распространяется вдоль поверхности металла и локализуется вблизи его поверхности [2,3], поэтому даже ничтожные изменения показателя преломления в приповерхностной области сильно влияют на характер ее распространения. На этом основывается широкое использование схемы Кречмана для создания различного рода высокочувствительных датчиков, реагирующих на изменение показателя преломления тонкого (олиго- или мономолекулярного) поверхностного слоя [4].

Исследования показали [5], что амплитуда поверхностной волны на свободной границе пленки в схеме Кречмана более чем на порядок выше амплитуды падающей волны. Так как в свободном пространстве над пленкой не возбуждаются распространяющиеся волны, это дает возможность, помещая на свободную поверхность наночастицы или отдельные молекулы, наблюдать исключительно их излучение, направленное в сторону свободного полупространства. Это излучение порождается наведенными электрическими дипольными моментами наночастиц, которые индуцируются поверхностной волной. Важно, что только точечные объекты будут излучать распространяющиеся волны в свободное пространство, и исключительно это излучение можно будет наблюдать с помощью микроскопа, при этом можно полностью быть уверенным, что наблюдаемый в микро- скоп свет исходит именно из точечных источников на поверхности. Подобный метод подсветки микрочастиц экспоненциально убывающей волной, возникающей при полном внутреннем отражении, применялся при исследовании коллоидных растворов Э. Коттоном и А. Мутоном с помощью усовершенствованного ультрамикроскопа [6]. Однако подсветка поверхностной плазмонной волной на поверхности металлической пленки кажется более перспективной по многим причинам, в частности при таком способе уменьшается количество паразитных переотражений в системе, исключается неудобство подсветки под очень малыми углами с внутренней стороны призмы, и этот способ может применяться совместно с измерениями известными датчиками из-

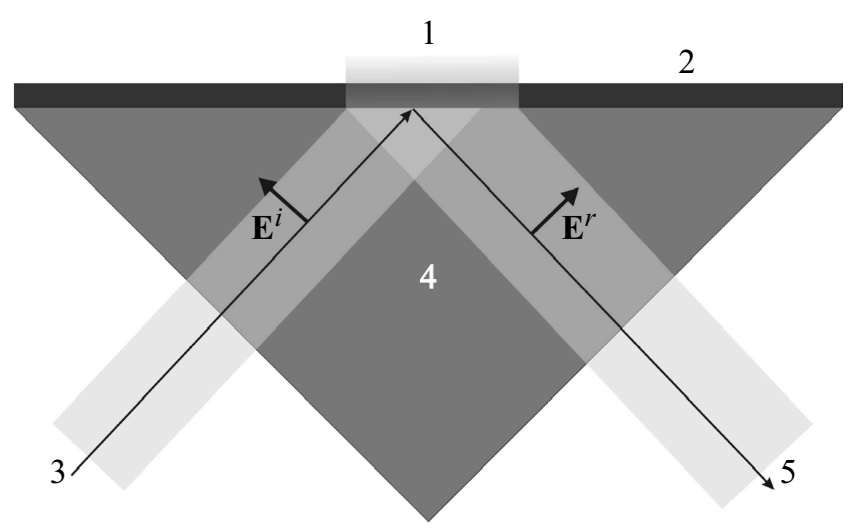

Рис. 1. Возбуждение поверхностной плазмонной волны 1 на поверхности металлической пленки 2 по схеме Кречмана. Падающая $p$-поляризованная волна 3 со стороны призмы 4 порождает на свободной границе поверхностную плазмонную 1 и отраженную 5 волны. 
менения показателя преломления вблизи поверхности пленки.

В связи с этим возникает фундаментальный вопрос о том, как влияет металлическая пленка (или в общем случае многопленочная структура) в схеме Кречмана на пространственное излучение в свободное пространство над пленкой от точечного излучателя, расположенного на поверхности. Теоретические методы нахождения излучения от элементарного точечного электрического диполя, расположенного на плоской границе двух сред, были развиты на заре эры использования электромагнитных волн для передачи сигналов вдоль земной поверхности [7]. В дальнейшем эти методы получили дальнейшее развитие в связи с возникающими новыми задачами радиосвязи $[8,9]$. Развитие антенной техники привело к развитию теории излучения элементарного диполя, расположенного на границе плоскослоистой среды, и теории излучения микрополосковых антенн [10-12], которые являются наилучшими конструкциями с точки зрения их совместимости с микрополосковыми интегральными схемами. Такие антенны играют особую роль в современной технике, так как они могут быть изготовлены из фольгированных материалов высокопроизводительными методами печати и травления.

Существующие теоретические методы расчета излучения антенн, расположенных на границе слоистых структур, сложны и описываются, например, в терминах диадных функций Грина [13]. Кроме того, сама теория распространения волн в слоистых структурах содержит много тонких моментов, таких как необходимость правильного выбора ветвей аналитических функций при записи волн в слоях, которые могут приводить к чудесам вроде суперразрешения [14].

В работах $[15,16]$ был предложен вариант строгой электромагнитной теории излучения элементарного диполя, расположенного на границе или внутри плоскослоистой структуры. Для случаев излучения горизонтального и вертикального диполей, расположенных на свободной границе одной пленки, был продемонстрирован метод аналитического упрощения решения, имеющий, как кажется, потенциально важное общетеоретическое значение. Этот метод позволил привести формулы для излучаемых полей к одномерным интегралам, что существенно упростило анализ задачи и ускорило численные расчеты.

Настоящая работа является продолжением работ $[15,16]$. Как будет показано ниже, формулы для полей в дальней зоне диполей в сторону полупространств вокруг плоскослоистой структуры допускают существенное упрощение не только для плоскослоистой структуры, состоящей из одной пленки, но и для сложной многопленочной структуры.

\section{Постановка задачи. Излучение элементарного излучателя, расположенного внутри плоскослоистой структуры}

Рассмотрим задачу излучения электромагнитной волны точечным источником тока единичной амплитуды, изменяющимся во времени гармонически с циклической частотой $\omega$. Пусть этот источник расположен в плоской слоистой структуре, состоящей из нескольких пленок, и из (окружающих слоистую структуру) двух полупространств. Для определенности сначала будем считать, что источник расположен внутри одной из пленок, а затем обобщим эту задачу на случай, когда источник расположен на их границе.

Пусть общее число пленок равно $N_{f}$, толщина $m$-й пленки равна $d_{m}$ и полная толщина слоистой структуры равна $d_{\text {tot }}=\sum_{m=1}^{N_{f}} d_{m}$. Общее число границ между пленками обозначим как $N=N_{f}+1$. Пронумеруем области пространства $j=1, \ldots,(N+1)$ (на рис. 2 показана для примера задача с $N=4$ и $\left.N_{f}=3\right)$. Предположим, что пленки имеют абсолютные комплексные диэлектрические и магнитные проницаемости, равные $\varepsilon_{j}$ и $\mu_{j}$ на рассматриваемой частоте $\omega$, а перед и за слоистой структурой находятся однородные полупространства с проницаемостями $\varepsilon_{1}, \mu_{1}$ и $\varepsilon_{N+1}, \mu_{N+1}$ (свободное пространство). Обозначим также через $z_{j}$ координаты $N$ границ пленок по оси $Z$ следующим образом: $z_{1}=0$, $z_{j}=\sum_{m=1}^{j-1} d_{m}$ при $j=2, \ldots, N$.

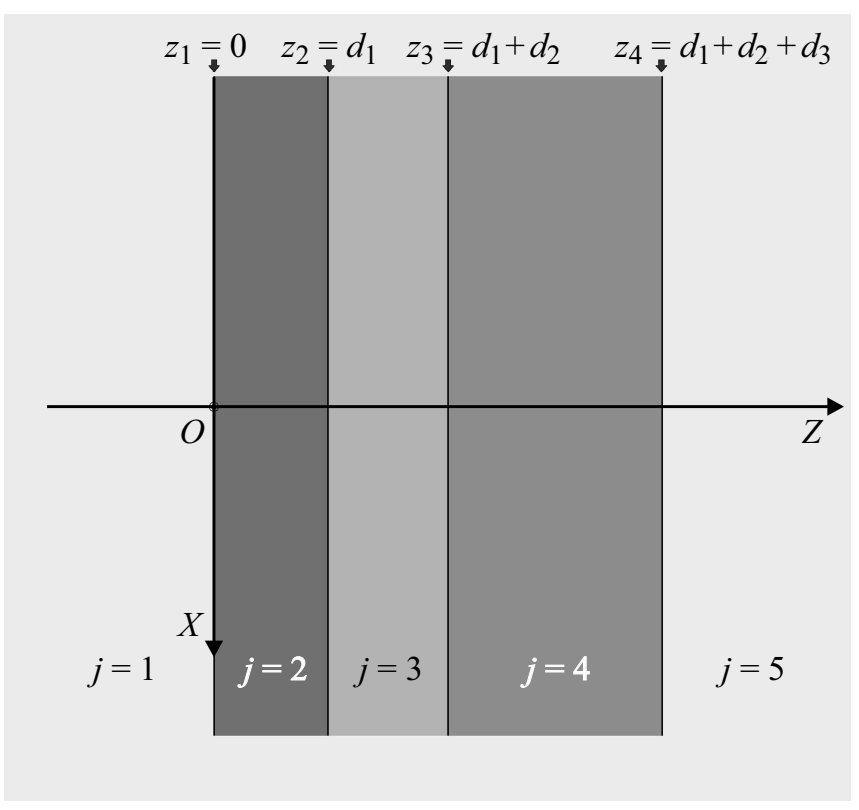

Рис. 2. Геометрия плоскослоистой структуры, состоящей из трех пленок. 


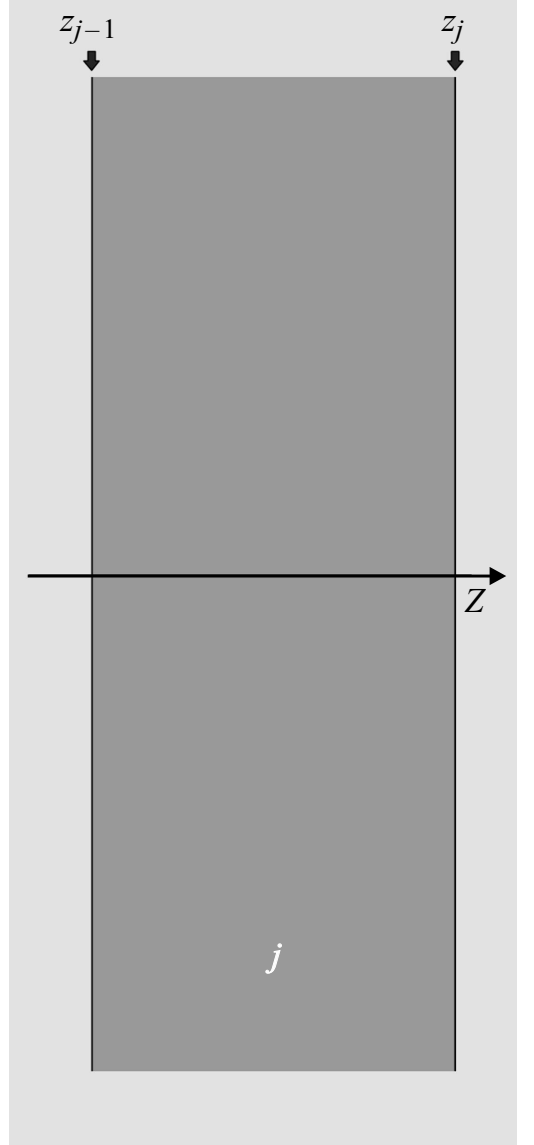

Рис. 3. Пленка с номером $j$, расположенная между границами $z_{j-1}$ и $z_{j}$

Уравнения Максвелла для области с номером $j$ можно записать в виде

$$
\begin{gathered}
\operatorname{rot} \mathbf{E}_{j}=i \omega \mathbf{B}_{j}, \\
\operatorname{rot} \mathbf{B}_{j}=\mu_{j}\left(-i \omega \varepsilon_{j} \mathbf{E}_{j}+\mathbf{J}_{j}\right),
\end{gathered}
$$

где $\mathbf{E}_{j}, \mathbf{B}_{j}$ и $\mathbf{J}_{j}-$ векторы напряженности электрического поля, индукция магнитного поля и плотность стороннего (известного) тока в области с номером $j$ (если источник тока отсутствует в области $j$, то $\mathbf{J}_{j}=0$ ). Предполагается комплексное временное представление в виде $e^{-i \omega t}$.

Решая уравнения Максвелла в каждой области с учетом граничных условий, найдем электромагнитное поле во всех областях. Рассмотрим сначала следующую вспомогательную задачу.

\section{Распространение электромагнитной волны в слое, свободном от сторонних токов}

Пусть в области с номером $j$ нет сторонних токов между границами $z_{j-1}$ и $z_{j}$ (рис. 3). Диэлектрическая и магнитная проницаемости среды в этой пленке равны $\varepsilon_{j}$ и $\mu_{j}$ соответственно. Тогда, учитывая, что в этой области справедливо уравнение $\operatorname{div} \mathbf{D}_{j}=0$, из уравнений (1) и (2) получаем уравнения для электромагнитных полей в виде

$$
\begin{aligned}
& \operatorname{rot} \operatorname{rot} \mathbf{E}_{j}-\omega^{2} \varepsilon_{j} \mu_{j} \mathbf{E}_{j}=0, \\
& \operatorname{rot} \operatorname{rot} \mathbf{B}_{j}-\omega^{2} \varepsilon_{j} \mu_{j} \mathbf{B}_{j}=0 .
\end{aligned}
$$

В рассматриваемой области $\operatorname{div} \mathbf{E}_{j}=0 \quad$ и $\operatorname{div} \mathbf{B}_{j}=0 . \quad$ Учитывая векторное тождество $\operatorname{rot} \operatorname{rot} \mathbf{F}=\operatorname{grad} \operatorname{div} \mathbf{F}-\Delta \mathbf{F}, \quad$ где $\quad \Delta=\partial_{x x}^{2}+\partial_{y y}^{2}+\partial_{z z}^{2}-$ оператор Лапласа, из (3) и (4) получим

$$
\begin{aligned}
& \Delta \mathbf{E}_{j}+\omega^{2} \varepsilon_{j} \mu_{j} \mathbf{E}_{j}=0, \\
& \Delta \mathbf{B}_{j}+\omega^{2} \varepsilon_{j} \mu_{j} \mathbf{B}_{j}=0 .
\end{aligned}
$$

Подставим в полученные выше уравнения компоненты полей в виде фурье-разложений. Например, представление для $x$-компоненты электрического поля примет вид

$$
E_{j, x}(x, y, z)=\frac{1}{(2 \pi)^{2}} \int_{-\infty}^{+\infty} \int_{-\infty}^{+\infty} \tilde{E}_{j, x}(\xi, \eta, z) e^{i(\xi x+\eta y)} d \xi d \eta
$$

где фурье-образ определяется выражением

$$
\tilde{E}_{j, x}(\xi, \eta, z)=\int_{-\infty}^{+\infty} \int_{-\infty}^{+\infty} E_{j, x}(x, y, z) e^{-i(\xi x+\eta y)} d x d y
$$

Для остальных компонент полей будем использовать аналогичные представления и соответствующие символы.

В компонентах уравнения (5) и (6) имеют вид

$$
\partial_{x x}^{2} E_{j, x}+\partial_{y y}^{2} E_{j, x}+\partial_{z z}^{2} E_{j, x}+\omega^{2} \mu_{j} \varepsilon_{j} E_{j, x}=0,
$$

и аналогичные уравнения для $E_{j, y}, E_{j, z}$ и составляющих магнитного поля, которые выписывать не будем. Переходя к фурье-образам, получаем из (5) три уравнения

$$
\begin{gathered}
\frac{d^{2} \tilde{E}_{j, x}}{d z^{2}}+\gamma_{j}^{2} \tilde{E}_{j, x}=0 ; \quad \frac{d^{2} \tilde{E}_{j, y}}{d z^{2}}+\gamma_{j}^{2} \tilde{E}_{j, y}=0 ; \\
\frac{d^{2} \tilde{E}_{j, z}}{d z^{2}}+\gamma_{j}^{2} \tilde{E}_{j, z}=0,
\end{gathered}
$$

где $\gamma_{j}=\sqrt{k_{j}^{2}-\xi^{2}-\eta^{2}}, k_{j}=\omega \sqrt{\mu_{j} \varepsilon_{j}}$.

Решения уравнений (7) для направлений распространения волн вдоль „+““ и против „-“ оси Z можно записать в виде

$$
\mathbf{E}_{j}^{ \pm}(x, y, z)=\frac{1}{(2 \pi)^{2}} \int_{-\infty}^{+\infty} \int_{-\infty}^{+\infty}\left(\begin{array}{c}
\hat{E}_{j, x}^{ \pm} \\
\hat{E}_{j, y}^{ \pm} \\
\hat{E}_{j, z}^{ \pm}
\end{array}\right) e^{ \pm i \gamma_{j} z} e^{i(\xi x+\eta y)} d \xi d \eta
$$


В рассматриваемом случае $\operatorname{div} \mathbf{E}_{j}=0$ и, следовательно, $\xi \hat{E}_{j, x}^{ \pm}+\eta \hat{E}_{j, y}^{ \pm} \pm \gamma_{j} \hat{E}_{j, z}^{ \pm}=0$. Тогда (8) можно переписать в виде

$$
\begin{aligned}
\mathbf{E}_{j}^{ \pm}(x, y, z)= & \frac{1}{(2 \pi)^{2}} \int_{-\infty}^{+\infty} \int_{-\infty}^{+\infty}\left(\begin{array}{cc}
1 & 0 \\
0 & 1 \\
\mp \xi / \gamma_{j} & \mp \eta / \gamma_{j}
\end{array}\right)\left(\begin{array}{c}
\hat{E}_{j, x}^{ \pm} \\
\hat{E}_{j, y}^{ \pm}
\end{array}\right) \\
& \times e^{ \pm i \gamma_{j} z} e^{i(\xi x+\eta y)} d \xi d \eta .
\end{aligned}
$$

Поэтому общее решение уравнений (7) в области $\left[z_{j-1}, z_{j}\right]$ можно записать в виде

$$
\begin{aligned}
& \mathbf{E}_{j}(x, y, z)=\frac{1}{(2 \pi)^{2}} \int_{-\infty}^{+\infty} \int_{-\infty}^{+\infty}\left(\begin{array}{cc}
1 & 0 \\
0 & 1 \\
-\xi / \gamma_{j} & -\eta / \gamma_{j}
\end{array}\right)\left(\begin{array}{c}
\hat{E}_{j, x}^{+} \\
\hat{E}_{j, y}^{+}
\end{array}\right) \\
& \times e^{i \gamma_{j}\left(z-z_{j-1}\right)} e^{i(\xi x+\eta y)} d \xi d \eta+ \\
& +\frac{1}{(2 \pi)^{2}} \int_{-\infty}^{+\infty} \int_{-\infty}^{+\infty}\left(\begin{array}{cc}
1 & 0 \\
0 & 1 \\
\xi / \gamma_{j} & \eta / \gamma_{j}
\end{array}\right)\left(\begin{array}{c}
\hat{E}_{j, x}^{-} \\
\hat{E}_{j, y}^{-}
\end{array}\right) e^{-i \gamma_{j}\left(z-z_{j}\right)} \\
& \times e^{i(\xi x+\eta y)} d \xi d \eta .
\end{aligned}
$$

Обратим внимание на отличие в форме записи (9) и (10). Формально эти уравнения переходят одно в другое; они описывают волны, распространяющиеся в противоположных направлениях по оси $Z$. Однако формулы содержат функции $\gamma_{j}=\sqrt{k_{j}^{2}-\xi^{2}-\eta^{2}}$. Для однозначного определения вида записи решений необходимо однозначно выбрать аналитическую ветвь функции комплексного переменного $\gamma_{j}(\lambda)$, где $\lambda^{2}=\xi^{2}+\eta^{2}$.

Обычно для сред без поглощения используют ветвь [9]

$$
\gamma_{j}(\lambda)= \begin{cases}\sqrt{k_{j}^{2}-\lambda^{2}}, & \lambda^{2} \leq k_{j}^{2}, \\ i \sqrt{\lambda^{2}-k_{j}^{2}}, & \lambda^{2} \geq k_{j}^{2} .\end{cases}
$$

Если взять вместо (11) другую аналитическую ветвь - с отрицательной мнимой зависимостью, то волны с большими $\lambda$ будут экспоненциально возрастать $\mathrm{c} z$ при удалении от источников полей, что противоречит принципу причинности.

Более строго, в случае поглощающей среды существует две точки ветвления функции $\gamma_{j}(\lambda)$ : точка $k_{j, 1}=\omega \sqrt{\left|\varepsilon_{j}\right|\left|\mu_{j}\right|} \exp \left(i\left(\arg \left(\varepsilon_{j}\right)+\arg \left(\mu_{j}\right)\right) / 2\right)$ и точка $k_{j, 2}=e^{i \pi} k_{j, 1}$. Аналитическую ветвь функции $\gamma_{j}(\lambda)$, пригодную в том числе для описания материалов с отрицательным преломлением [17] и переходящую в (11) при бесконечно малом поглощении среды, можно определить как

$$
\begin{aligned}
\gamma_{j}(\lambda)= & \sqrt{\left|k_{j, 1}-\lambda\right|} \exp \left(\frac{i \arg \left(k_{j, 1}-\lambda\right)}{2}\right) \\
& \times \sqrt{\left|k_{j, 2}-\lambda\right|} \exp \left(\frac{i \arg \left(\lambda-k_{j, 2}\right)}{2}\right),
\end{aligned}
$$

где функции $|\xi|$ и $\arg (\xi)$ есть модуль и аргумент комплексной переменной $\xi$.

В представлении полей (10) при любых $z$ и правильном выборе аналитической ветви (12) будет обеспечена сходимость интегралов. При этом не будут возникать экспоненциально усиливающиеся гармоники при больших значениях $\xi$ и $\eta$.

Из уравнения $\operatorname{rot} \mathbf{E}_{j}=i \omega \mathbf{B}_{j}$ найдем $x$ - и $y$-компоненты магнитного поля. Для соответствующих фурьекомпонент находим

$$
\begin{aligned}
\hat{\mathbf{B}}_{j}^{+}= & \mathbf{e}_{x}\left(\frac{\eta}{\omega} \hat{E}_{j, z}^{+}-\frac{\gamma_{j}}{\omega} \hat{E}_{j, y}^{+}\right)-\mathbf{e}_{y}\left(\frac{\xi}{\omega} \hat{E}_{j, z}^{+}-\frac{\gamma_{j}}{\omega} \hat{E}_{j, x}^{+}\right) \\
& +\mathbf{e}_{z}\left(\frac{\xi}{\omega} \hat{E}_{j, y}^{+}-\frac{\eta}{\omega} \hat{E}_{j, x}^{+}\right),
\end{aligned}
$$

где $\mathbf{e}_{x}, \mathbf{e}_{y}$ и $\mathbf{e}_{z}-$ единичные орты координатных осей.

Из $\operatorname{div} \mathbf{E}_{j}=0$ следует $\hat{E}_{j, z}^{+}=-\xi \hat{E}_{j, x}^{+} / \gamma_{j}-\eta \hat{E}_{j, y}^{+} / \gamma_{j}$, тогда

$$
\begin{aligned}
& \hat{\mathbf{B}}_{j}^{+}=\mathbf{e}_{x}\left(-\frac{\xi \eta}{\omega \gamma_{j}} \hat{E}_{j, x}^{+}-\left(\frac{\gamma_{j}^{2}+\eta^{2}}{\omega \gamma_{j}}\right) \hat{E}_{j, y}^{+}\right) \\
& +\mathbf{e}_{y}\left(\left(\frac{\gamma_{j}^{2}+\xi^{2}}{\omega \gamma_{j}}\right) \hat{E}_{j, x}^{+}+\frac{\xi \eta}{\omega \gamma_{j}} \hat{E}_{j, y}^{+}\right)+\mathbf{e}_{z}\left(\frac{\xi}{\omega} \hat{E}_{j, y}^{+}-\frac{\eta}{\omega} \hat{E}_{j, x}^{+}\right),
\end{aligned}
$$

или в матричном виде

$$
\left(\begin{array}{c}
\hat{B}_{j, x}^{+} \\
\hat{B}_{j, y}^{+} \\
\hat{B}_{j, z}^{+}
\end{array}\right)=\left(\begin{array}{cc}
-\xi \eta / \omega \gamma_{j} & -\left(\gamma_{j}^{2}+\eta^{2}\right) / \omega \gamma_{j} \\
\left(\gamma_{j}^{2}+\xi^{2}\right) / \omega \gamma_{j} & \xi \eta / \omega \gamma_{j} \\
-\eta / \omega & \xi / \omega
\end{array}\right)\left(\begin{array}{c}
\hat{E}_{j, x}^{+} \\
E_{j, y}^{+}
\end{array}\right) .
$$

Аналогично для $\hat{\mathbf{B}}_{j}^{-}$найдем

$$
\left(\begin{array}{c}
\hat{B}_{j, x}^{-} \\
\hat{B}_{j, y}^{-} \\
\hat{B}_{j, z}^{-}
\end{array}\right)=\left(\begin{array}{cc}
\xi \eta / \omega \gamma_{j} & \left(\gamma_{j}^{2}+\eta^{2}\right) / \omega \gamma_{j} \\
-\left(\gamma_{j}^{2}+\xi^{2}\right) / \omega \gamma_{j} & -\xi \eta / \omega \gamma_{j} \\
-\eta / \omega & \xi / \omega
\end{array}\right)\left(\begin{array}{c}
\hat{E}_{j, x}^{-} \\
\hat{E}_{j, y}^{-}
\end{array}\right) .
$$

Тогда общее решение для магнитного поля в рассматриваемой области с номером $j$ (пленке с номером $j-1$ ) можно записать в виде

$$
\begin{aligned}
& \mathbf{B}_{j}(x, y, z)= \\
& =\frac{1}{(2 \pi)^{2}} \int_{-\infty}^{+\infty} \int_{-\infty}^{+\infty}\left(\begin{array}{cc}
-\xi \eta / \omega \gamma_{j} & -\left(\gamma_{j}^{2}+\eta^{2}\right) / \omega \gamma_{j} \\
\left(\gamma_{j}^{2}+\xi^{2}\right) / \omega \gamma_{j} & \xi \eta / \omega \gamma_{j} \\
-\eta / \omega & \xi / \omega
\end{array}\right) \\
& \times\left(\begin{array}{c}
\hat{E}_{j, x}^{+} \\
\hat{E}_{j, y}^{+}
\end{array}\right) e^{i \gamma_{j}\left(z-z_{j-1}\right)} e^{i(\xi x+\eta y)} d \xi d \eta+ \\
& +\frac{1}{(2 \pi)^{2}} \int_{-\infty-\infty}^{+\infty} \int_{-\infty}^{+\infty}\left(\begin{array}{cc}
\xi \eta / \omega \gamma_{j} & \left(\gamma_{j}^{2}+\eta^{2}\right) / \omega \gamma_{j} \\
-\left(\gamma_{j}^{2}+\xi^{2}\right) / \omega \gamma_{j} & -\xi \eta / \omega \gamma_{j} \\
-\eta / \omega & \xi / \omega
\end{array}\right) \\
& \times\left(\begin{array}{c}
\hat{E}_{j, x}^{-} \\
\hat{E}_{j, y}^{-}
\end{array}\right) e^{-i \gamma_{j}\left(z-z_{j}\right)} e^{i(\xi x+\eta y)} d \xi d \eta .
\end{aligned}
$$


Из (10) и (13) найдем тангенциальные составляющие фурье-образов полей на границах области $j$ в виде

$$
\begin{aligned}
& \left.\left(\begin{array}{c}
\tilde{E}_{j, x} \\
\tilde{E}_{j, y} \\
\tilde{B}_{j, x} / \mu_{j} \\
\tilde{B}_{j, y} / \mu_{j}
\end{array}\right)\right|_{z=z_{j-1}} \\
& =\left(\begin{array}{cccc}
1 & 0 & e^{i \gamma_{j} d_{j-1}} & 0 \\
0 & 1 & 0 & e^{i \gamma_{j} d_{j-1}} \\
-\frac{\xi \eta}{\omega \mu_{j} \gamma_{j}} & -\frac{\left(\gamma_{j}^{2}+\eta^{2}\right)}{\omega \mu_{j} \gamma_{j}} & \frac{\xi \eta}{\omega \mu_{j} \gamma_{j}} e^{i \gamma_{j} d_{j-1}} & \frac{\left(\gamma_{j}^{2}+\eta^{2}\right)}{\omega \mu_{j} \gamma_{j}} e^{i \gamma_{j} d_{j-1}} \\
\frac{\left(\gamma_{j}^{2}+\xi^{2}\right)}{\omega \mu_{j} \gamma_{j}} & \frac{\xi \eta}{\omega \mu_{j} \gamma_{j}} & -\frac{\left(\gamma_{j}^{2}+\xi^{2}\right)}{\omega \mu_{j} \gamma_{j}} e^{i \gamma_{j} d_{j-1}} & -\frac{\xi \eta}{\omega \mu_{j} \gamma_{j}} e^{i \gamma_{j} d_{j-1}}
\end{array}\right) \\
& \times\left(\begin{array}{c}
\hat{E}_{j, x}^{+} \\
\hat{E}_{j, y}^{+} \\
\hat{E}_{j, x}^{-} \\
\hat{E}_{j, y}^{-}
\end{array}\right),
\end{aligned}
$$$$
\left.\left(\begin{array}{c}
\tilde{E}_{j, x} \\
\tilde{E}_{j, y} \\
\tilde{B}_{j, x} / \mu_{j} \\
\tilde{B}_{j, y} / \mu_{j}
\end{array}\right)\right|_{z=z_{j}}=
$$$$
=\left(\begin{array}{cccc}
e^{i \gamma_{j} d_{j-1}} & 0 & 1 & 0 \\
0 & e^{i \gamma_{j} d_{j-1}} & 0 & 1 \\
-\frac{\xi \eta}{\omega \mu_{j} \gamma_{j}} e^{i \gamma_{j} d_{j-1}} & -\frac{\left(\gamma_{j}^{2}+\eta^{2}\right)}{\omega \mu_{j} \gamma_{j}} e^{i \gamma_{j} d_{j-1}} & \frac{\xi \eta}{\omega \mu_{j} \gamma_{j}} & \frac{\left(\gamma_{j}^{2}+\eta^{2}\right)}{\omega \mu_{j} \gamma_{j}} \\
\frac{\left(\gamma_{j}^{2}+\xi^{2}\right)}{\omega \mu_{j} \gamma_{j}} e^{i \gamma_{j} d_{j-1}} & \frac{\xi \eta}{\omega \mu_{j} \gamma_{j}} e^{i \gamma_{j} d_{j-1}} & -\frac{\left(\gamma_{j}^{2}+\xi^{2}\right)}{\omega \mu_{j} \gamma_{j}} & -\frac{\xi \eta}{\omega \mu_{j} \gamma_{j}}
\end{array}\right)
$$$$
\times\left(\begin{array}{c}
\hat{E}_{j, x}^{+} \\
\hat{E}_{j, y}^{+} \\
\hat{E}_{j, x}^{-} \\
\hat{E}_{j, y}^{-}
\end{array}\right),
$$

где $d_{j-1}=z_{j}-z_{z-1}$.

Вводя вектор-столбец $\hat{\mathfrak{E}}_{j}=\left(\hat{E}_{j, x}^{+} ; \hat{E}_{j, y}^{+} ; \hat{E}_{j, x}^{-} ; \hat{E}_{j, y}^{-}\right)^{T}$, запишем полученные выражения в матричном виде:

$$
\begin{aligned}
& \left.\left(\begin{array}{c}
\tilde{E}_{j, x} \\
\tilde{E}_{j, y} \\
\tilde{B}_{j, x} / \mu_{j} \\
\tilde{B}_{j, y} / \mu_{j}
\end{array}\right)\right|_{z=z_{j-1}}=\left(\begin{array}{cc}
\mathbf{I} & e^{i \gamma_{j} d_{j-1} \mathbf{I}} \\
\mathbf{G}_{j} & -e^{i \gamma_{j} d_{j-1}} \mathbf{G}_{j}
\end{array}\right) \hat{\mathfrak{E}}_{j}, \\
& \left.\left(\begin{array}{c}
\tilde{E}_{j, x} \\
\tilde{E}_{j, y} \\
\tilde{B}_{j, x} / \mu_{j} \\
\tilde{B}_{j, y} / \mu_{j}
\end{array}\right)\right|_{z=z_{j}}=\left(\begin{array}{cc}
e^{i \gamma_{j} d_{j-1} \mathbf{I}} & \mathbf{I} \\
e^{i \gamma_{j} d_{j-1}} \mathbf{G}_{j} & -\mathbf{G}_{j}
\end{array}\right) \hat{\mathfrak{E}}_{j},
\end{aligned}
$$

где $\mathbf{I}-$ единичная $2 \times 2$-матрица, а матрица $\mathbf{G}_{j}$ имеет вид

$$
\mathbf{G}_{j}=\left(\begin{array}{cc}
-\frac{\xi \eta}{\omega \mu_{j} \gamma_{j}} & -\frac{\gamma_{j}^{2}+\eta^{2}}{\omega \mu_{j} \gamma_{j}} \\
\frac{\gamma_{j}^{2}+\xi^{2}}{\omega \mu_{j} \gamma_{j}} & \frac{\xi \eta}{\omega \mu_{j} \gamma_{j}}
\end{array}\right)
$$

\section{Распространение электромагнитной волны в многослойной структуре, свободной от сторонних токов}

Рассмотрим теперь многослойную структуру, внутри которой нет сторонних токов. Рассмотрим границу $z=z_{j}$ между областями с номерами $j$ и $j+1$. Непрерывность тангенциальных компонент напряженностей электрического и магнитного полей на этой границе можно записать в виде

$$
\begin{gathered}
E_{j, x}\left(x, y, z_{j}\right)-E_{j+1, x}\left(x, y, z_{j}\right)=0, \\
E_{j, y}\left(x, y, z_{j}\right)-E_{j+1, y}\left(x, y, z_{j}\right)=0, \\
B_{j, x}\left(x, y, z_{j}\right) / \mu_{j}-B_{j+1, x}\left(x, y, z_{j}\right) / \mu_{j+1}=0, \\
B_{j, y}\left(x, y, z_{j}\right) / \mu_{j}-B_{j+1, y}\left(x, y, z_{j}\right) / \mu_{j+1}=0,
\end{gathered}
$$

где электрические и магнитные поля в области $j+1$ выражаются формулами (10) и (13), в которых произведена замена индексов $j \rightarrow j+1$. Так как уравнения Максвелла - линейные уравнения, то граничные условия должны выполняться для каждого члена фурьеразложения, т.е. граничные условия выполняются для фурье-образов полей:

$$
\begin{gathered}
\tilde{E}_{j, x}\left(\xi, \eta, z_{j}\right)-\tilde{E}_{j+1, x}\left(\xi, \eta, z_{j}\right)=0, \\
\tilde{E}_{j, y}\left(\xi, \eta, z_{j}\right)-\tilde{E}_{j+1, y}\left(\xi, \eta, z_{j}\right)=0, \\
\tilde{B}_{j, x}\left(\xi, \eta, z_{j}\right) / \mu_{j}-\tilde{B}_{j+1, x}\left(\xi, \eta, z_{j}\right) / \mu_{j+1}=0, \\
\tilde{B}_{j, y}\left(\xi, \eta, z_{j}\right) / \mu_{j}-\tilde{B}_{j+1, y}\left(\xi, \eta, z_{j}\right) / \mu_{j+1}=0 .
\end{gathered}
$$

Записывая граничные условия (17)-(20) с помощью выражений (14) и (15), получим матричное уравнение на границе $z=z_{j}$ :

$$
\left(\begin{array}{cc}
e^{i \gamma_{j} d_{j-1} \mathbf{I}} & \mathbf{I} \\
e^{i \gamma_{j} d_{j-1}} \mathbf{G}_{j} & -\mathbf{G}_{j}
\end{array}\right) \hat{\mathfrak{E}}_{j}=\left(\begin{array}{cc}
\mathbf{I} & e^{i \gamma_{j+1} d_{j} \mathbf{I}} \\
\mathbf{G}_{j+1} & -e^{i \gamma_{j+1} d_{j}} \mathbf{G}_{j+1}
\end{array}\right) \hat{\mathfrak{E}}_{j+1},
$$

где $d_{j-1}=z_{j}-z_{j-1}, d_{j}=z_{j+1}-z_{j}$.

Уравнение (21) можно записать для $j=2, \ldots,(N-1)$, где $(N+1)$ - общее число областей, $N$ - число границ, т.е. для всех границ, исключая первую $(j=1)$ и последнюю $(j=N)$ границы. То есть исключая границу $z_{1}=0$ и $z_{N}=d_{\text {tot }}=\sum_{m=1}^{N-1} d_{m}$, где $d_{\text {tot }}$ - общая толщина слоистой структуры (сумма толщин пленок, составляющих рассматриваемую структуру).

Общее решение для электрического и магнитного полей в области $j=1$, т.е. в интервале $\left(-\infty, z_{1}\right)$, где 
$z_{1}=0$, запишем в виде

$$
\begin{aligned}
& \mathbf{E}_{1}(x, y, z)=\frac{1}{(2 \pi)^{2}} \int_{-\infty}^{+\infty} \int_{-\infty}^{+\infty}\left(\begin{array}{cc}
1 & 0 \\
0 & 1 \\
-\xi / \gamma_{1} & -\eta / \gamma_{1}
\end{array}\right) \\
& \times\left(\begin{array}{c}
\hat{E}_{1, x}^{+} \\
\hat{E}_{1, y}^{+}
\end{array}\right) e^{i \gamma_{1} z} e^{i(\xi x+\eta y)} d \xi d \eta+ \\
& +\frac{1}{(2 \pi)^{2}} \int_{-\infty}^{+\infty} \int_{-\infty}^{+\infty}\left(\begin{array}{cc}
1 & 0 \\
0 & 1 \\
\xi / \gamma_{1} & \eta / \gamma_{1}
\end{array}\right)\left(\begin{array}{l}
\hat{E}_{1, x}^{-} \\
\hat{E}_{1, y}^{-}
\end{array}\right) \\
& \times e^{-i \gamma_{1} z} e^{i(\xi x+\eta y)} d \xi d \eta,
\end{aligned}
$$

$$
\begin{aligned}
& \mathbf{B}_{1}(x, y, z)= \\
& =\frac{1}{(2 \pi)^{2}} \int_{-\infty}^{+\infty} \int_{-\infty}^{+\infty}\left(\begin{array}{cc}
-\xi \eta / \omega \gamma_{1} & -\left(\gamma_{1}^{2}+\eta^{2}\right) / \omega \gamma_{1} \\
\left(\gamma_{1}^{2}+\xi^{2}\right) / \omega \gamma_{1} & \xi \eta / \omega \gamma_{1} \\
-\eta / \omega & \xi / \omega
\end{array}\right) \\
& \times\left(\begin{array}{c}
\hat{E}_{1, x}^{+} \\
\hat{E}_{1, y}^{+}
\end{array}\right) e^{i \gamma_{1} z} e^{i(\xi x+\eta y)} d \xi d \eta+ \\
& +\frac{1}{(2 \pi)^{2}} \int_{-\infty}^{+\infty} \int_{-\infty}^{+\infty}\left(\begin{array}{cc}
\xi \eta / \omega \gamma_{1} & \left(\gamma_{1}^{2}+\eta^{2}\right) / \omega \gamma_{1} \\
-\left(\gamma_{1}^{2}+\xi^{2}\right) / \omega \gamma_{1} & -\xi \eta / \omega \gamma_{1} \\
-\eta / \omega & \xi / \omega
\end{array}\right) \\
& \times\left(\begin{array}{c}
\hat{E}_{1, x}^{-} \\
\hat{E}_{1, y}^{-}
\end{array}\right) e^{-i \gamma_{1} z} e^{i(\xi x+\eta y)} d \xi d \eta \text {. }
\end{aligned}
$$

Тогда граничные условия на границе $z_{1}=0$ можно записать в виде

$$
\left(\begin{array}{cc}
\mathbf{I} & \mathbf{I} \\
\mathbf{G}_{1} & -\mathbf{G}_{1}
\end{array}\right) \hat{\mathfrak{E}}_{1}=\left(\begin{array}{cc}
\mathbf{I} & e^{i \gamma_{2} d_{1}} \mathbf{I} \\
\mathbf{G}_{2} & -e^{i \gamma_{2} d_{1}} \mathbf{G}_{2}
\end{array}\right) \hat{\mathfrak{E}}_{2} .
$$

Аналогично общее решение для электрического и магнитного полей в области $j=N+1$, т.е. в интервале $\left(z_{N},+\infty\right)$, запишем в виде

$$
\begin{aligned}
& \mathbf{E}_{N+1}(x, y, z)=\frac{1}{(2 \pi)^{2}} \int_{-\infty}^{+\infty} \int_{-\infty}^{+\infty}\left(\begin{array}{cc}
1 & 0 \\
0 & 1 \\
-\xi / \gamma_{N+1} & -\eta / \gamma_{N+1}
\end{array}\right) \\
& \times\left(\begin{array}{c}
\hat{E}_{N+1, x}^{+} \\
\hat{E}_{N+1, y}^{+}
\end{array}\right) e^{i \gamma_{N+1}\left(z-z_{N}\right)} e^{i(\xi x+\eta y)} d \xi d \eta+ \\
& +\frac{1}{(2 \pi)^{2}} \int_{-\infty}^{+\infty} \int_{-\infty}^{+\infty}\left(\begin{array}{cc}
1 & 0 \\
0 & 1 \\
\xi / \gamma_{N+1} & \eta / \gamma_{N+1}
\end{array}\right) \\
& \times\left(\begin{array}{c}
\hat{E}_{N+1, x}^{-} \\
\hat{E}_{N+1, y}^{-}
\end{array}\right) e^{-i \gamma_{N+1}\left(z-z_{N}\right)} e^{i(\xi x+\eta y)} d \xi d \eta,
\end{aligned}
$$

$$
\begin{aligned}
& \mathbf{B}_{N+1}(x, y, z)= \\
& =\frac{1}{(2 \pi)^{2}} \int_{-\infty-\infty}^{+\infty+\infty}\left(\begin{array}{cc}
-\xi \eta / \omega \gamma_{N+1} & -\left(\gamma_{N+1}^{2}+\eta^{2}\right) / \omega \gamma_{N+1} \\
\left(\gamma_{N+1}^{2}+\xi^{2}\right) / \omega \gamma_{N+1} & \xi \eta / \omega \gamma_{N+1} \\
-\eta / \omega & \xi / \omega
\end{array}\right) \\
& \times\left(\begin{array}{cc}
\hat{E}_{N+1, x}^{+} \\
\hat{E}_{N+1, y}^{+}
\end{array}\right) e^{i \gamma_{N+1}\left(z-z_{N}\right)} e^{i(\xi x+\eta y)} d \xi d \eta+ \\
& +\frac{1}{(2 \pi)^{2}} \int_{-\infty}^{+\infty} \int_{-\infty}^{\xi \eta / \omega \gamma_{N+1}}\left(\begin{array}{cc}
\left.\gamma_{N+1}^{2}+\eta^{2}\right) / \omega \gamma_{N+1} \\
-\left(\gamma_{N+1}^{2}+\xi^{2}\right) / \omega \gamma_{N+1} & -\xi \eta / \omega \gamma_{N+1} \\
-\eta / \omega & \xi / \omega
\end{array}\right) \\
& \times\left(\begin{array}{cc}
\hat{E}_{N+1, x}^{-} \\
\hat{E}_{N+1, y}^{-}
\end{array}\right) e^{-i \gamma_{N+1}\left(z-z_{N}\right)} e^{i(\xi x+\eta y)} d \xi d \eta .
\end{aligned}
$$

Тогда граничные условия на границе $z_{N}$ можно записать в виде

$$
\left(\begin{array}{cc}
e^{i \gamma_{N} d_{N-1} \mathbf{I}} & \mathbf{I} \\
e^{i \gamma_{N} d_{N-1}} \mathbf{G}_{N} & -\mathbf{G}_{N}
\end{array}\right) \hat{\mathfrak{E}}_{N}=\left(\begin{array}{cc}
\mathbf{I} & \mathbf{I} \\
\mathbf{G}_{N+1} & -\mathbf{G}_{N+1}
\end{array}\right) \hat{\mathfrak{E}}_{N+1} .
$$

Граничные условия (21), (24) и (27) позволяют связать векторы-столбцы электрического поля в первой и последней областях задачи (т.е. в полупространствах вне плоскослоистой структуры):

$$
\begin{aligned}
\hat{\mathfrak{E}}_{1}= & \left(\begin{array}{cc}
\mathbf{I} & \mathbf{I} \\
\mathbf{G}_{1} & -\mathbf{G}_{1}
\end{array}\right)^{-1} \times \\
\times & \left(\begin{array}{cc}
\mathbf{I} & e^{i \gamma_{2} d_{1} \mathbf{I}} \\
\mathbf{G}_{2} & -e^{i \gamma_{2} d_{1}} \mathbf{G}_{2}
\end{array}\right) \times\left(\begin{array}{cc}
e^{i \gamma_{2} d_{1} \mathbf{I}} & \mathbf{I} \\
e^{i \gamma_{2} d_{1}} \mathbf{G}_{2} & -\mathbf{G}_{2}
\end{array}\right)^{-1} \times \\
& \ldots\left(\begin{array}{cc}
\mathbf{I} & e^{i \gamma_{N} d_{N-1} \mathbf{I}} \\
\mathbf{G}_{N} & -e^{i \gamma_{N} d_{N-1}} \mathbf{G}_{N}
\end{array}\right) \times\left(\begin{array}{cc}
e^{i \gamma_{N} d_{N-1} \mathbf{I}} & \mathbf{I} \\
e^{i \gamma_{N} d_{N-1}} \mathbf{G}_{N} & -\mathbf{G}_{N}
\end{array}\right)^{-1} \\
& \times\left(\begin{array}{cc}
\mathbf{I} & \mathbf{I} \\
\mathbf{G}_{N+1} & -\mathbf{G}_{N+1}
\end{array}\right) \hat{\mathfrak{E}}_{N+1}
\end{aligned}
$$

или

$$
\hat{\mathfrak{E}}_{1}=\mathbf{M} \times \hat{\mathfrak{E}}_{N+1} .
$$

Матрица $\mathbf{M}$ имеет вид $\mathbf{M}=\mathbf{T}_{1} \times\left(\prod_{m=2}^{N} \mathbf{T}_{m}\right) \times \mathbf{T}_{N+1}$, где

$$
\begin{gathered}
\mathbf{T}_{1}=\left(\begin{array}{cc}
\mathbf{I} & \mathbf{I} \\
\mathbf{G}_{1} & -\mathbf{G}_{1}
\end{array}\right)^{-1} \\
\mathbf{T}_{m}=\left(\begin{array}{cc}
\mathbf{I} & e^{i \gamma_{m} d_{m-1} \mathbf{I}} \\
\mathbf{G}_{m} & -e^{i \gamma_{m} d_{m-1}} \mathbf{G}_{m}
\end{array}\right)\left(\begin{array}{cc}
e^{i \gamma_{m} d_{m-1} \mathbf{I}} & \mathbf{I} \\
e^{i \gamma_{m} d_{m-1}} \mathbf{G}_{m} & -\mathbf{G}_{m}
\end{array}\right)^{-1}, \\
\mathbf{T}_{N+1}=\left(\begin{array}{cc}
\mathbf{I} & \mathbf{I} \\
\mathbf{G}_{N+1} & -\mathbf{G}_{N+1}
\end{array}\right) .
\end{gathered}
$$

Если нам известна, например, падающая на плоскослоистую структуру волна, а значит, компоненты $\hat{E}_{1, x}^{+}$ и $\hat{E}_{1, y}^{+}$вектора-столбца $\hat{\mathfrak{E}}_{1}$, то из уравнения $(28)$ можно найти компоненты $\hat{E}_{1, x}^{-}$и $\hat{E}_{1, y}^{-}$и отраженную волну по формулам (22), (23), а также компоненты $\hat{E}_{N+1, x}^{+}$и $\hat{E}_{N+1, y}^{+}$ 


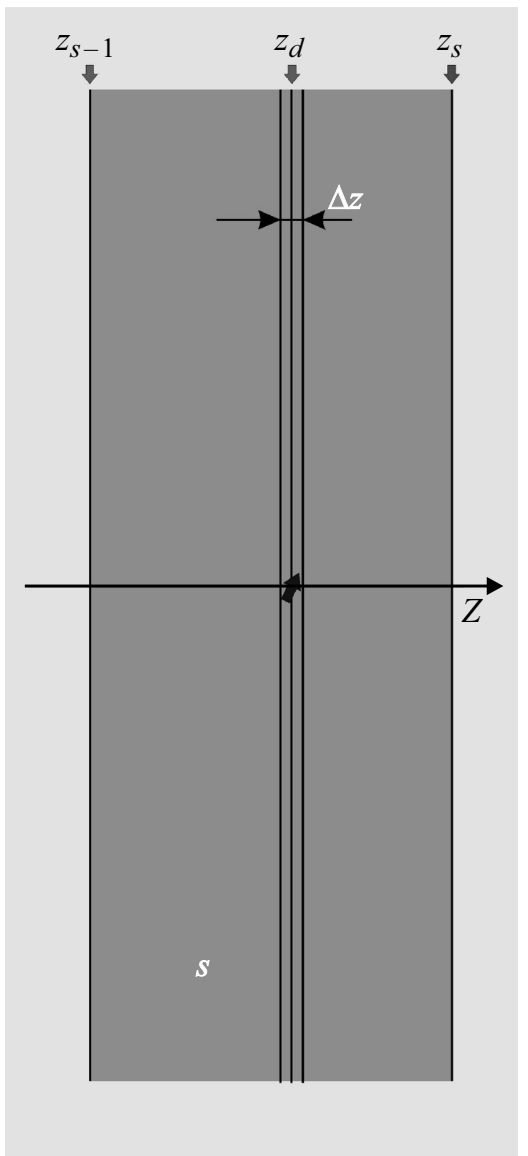

Рис. 4. Точечный излучатель, расположенный в точке $\left(0,0, z_{d}\right)$ в области с номером $s$.

вектора-столбца $\hat{\mathfrak{E}}_{N+1}$ и прошедшую волну по формулам (25), (26). Подробности решения подобных задач, в которых рассматриваются пространственно ограниченные падающие на плоскослоистую структуру пучки, можно найти, например, в работах [18-20].

\section{Распространение электромагнитной волны в многослойной структуре от элементарного источника тока}

Пусть имеется точечный излучатель, расположенный в точке $\left(0,0, z_{d}\right)$ в области с номером $s$ (рис. 4). Пусть этот излучатель определяется плотностью стороннего тока (в комплексном представлении $e^{-i \omega t}$ )

$$
\mathbf{J}(x, y, z)=\left(n_{x} \mathbf{e}_{x}+n_{y} \mathbf{e}_{y}+n_{z} \mathbf{e}_{z}\right) \delta(x) \delta(y) \delta\left(z-z_{d}\right),
$$

где $n_{x}, n_{y}, n_{z}$ - направляющие косинусы вектора тока вдоль осей координат, причем $n_{x}^{2}+n_{y}^{2}+n_{z}^{2}=1, \delta(x)-$ дельта-функция Дирака. Тогда фурье-образы составляю- щих этого тока определятся следующими выражениями:

$$
\begin{gathered}
\tilde{J}_{s, x}(\xi, \eta, z)=n_{x} \int_{-\infty}^{+\infty} \int_{-\infty}^{+\infty} \delta(x) \delta(y) \delta\left(z-z_{d}\right) \\
\times e^{-i(\xi x+\eta y)} d x d y=n_{x} \delta\left(z-z_{d}\right),
\end{gathered}
$$

и аналогично

$$
\begin{aligned}
& \tilde{J}_{s, y}(\xi, \eta, z)=n_{y} \delta\left(z-z_{d}\right), \\
& \tilde{J}_{s, z}(\xi, \eta, z)=n_{z} \delta\left(z-z_{d}\right) .
\end{aligned}
$$

Пусть этот элементарный источник тока находится в бесконечно тонком слое $\left(z_{d}-\Delta z / 2, z_{d}+\Delta z / 2\right)$. Тогда уравнения Максвелла (1) и (2) для фурье-образов полей можно записать (при $\Delta z \rightarrow 0$ ) в виде

$$
\begin{gathered}
\left\{\begin{array}{c}
i \eta \tilde{E}_{s, z}-\frac{\Delta \tilde{E}_{s, y}}{\Delta z}=i \omega \tilde{B}_{s, x}, \\
\frac{\Delta \tilde{E}_{s, x}}{\Delta z}-i \xi \tilde{E}_{s, z}=i \omega \tilde{B}_{s, y}, \\
i \xi \tilde{E}_{s, y}-i \eta \tilde{E}_{s, x}=i \omega \tilde{B}_{s, z}
\end{array}\right. \\
\left\{\begin{array}{c}
i \eta \tilde{B}_{s, z}-\frac{\Delta \tilde{B}_{j, y}}{\Delta z}=\mu_{s}\left(-i \omega \varepsilon_{s} \tilde{E}_{s, x}+n_{x} \delta\left(z-z_{d}\right)\right), \\
\frac{\Delta \tilde{B}_{s, x}}{\Delta z}-i \xi \tilde{B}_{s, z}=\mu_{s}\left(-i \omega \varepsilon_{s} \tilde{E}_{s, y}+n_{y} \delta\left(z-z_{d}\right)\right), \\
i \xi \tilde{B}_{s, y}-i \eta \tilde{B}_{s, x}=\mu_{s}\left(-i \omega \varepsilon_{s} \tilde{E}_{s, z}+n_{z} \delta\left(z-z_{d}\right)\right) .
\end{array}\right.
\end{gathered}
$$

Так как $z$-компоненты полей из уравнений (29) и (30) можно выразить через $x$ - и $y$-компоненты по формулам

$$
\begin{gathered}
\tilde{B}_{s, z}=\frac{\xi}{\omega} \tilde{E}_{s, y}-\frac{\eta}{\omega} \tilde{E}_{s, x}, \\
\tilde{E}_{s, z}=-\frac{\xi}{\omega \varepsilon_{s} \mu_{s}} \tilde{B}_{s, y}+\frac{\eta}{\omega \varepsilon_{s} \mu_{s}} \tilde{B}_{s, x}+\frac{1}{i \omega \varepsilon_{s}} \tilde{J}_{s, z},
\end{gathered}
$$

то для $x$ - и $y$-компонент напряженностей электрического и магнитного полей получим

$$
\left\{\begin{aligned}
\Delta \tilde{E}_{s, x}= & \left(\frac{i \xi \eta}{\omega \varepsilon_{s} \mu_{s}} \tilde{B}_{s, x}+i\left(\omega-\frac{\xi^{2}}{\omega \varepsilon_{s} \mu_{s}}\right) \tilde{B}_{s, y}\right. \\
& \left.+\frac{\xi}{\omega \varepsilon_{s}} n_{z} \delta\left(z-z_{d}\right)\right) \Delta z, \\
\Delta \tilde{E}_{s, y}= & \left(i\left(\frac{\eta^{2}}{\omega \varepsilon_{s} \mu_{s}}-\omega\right) \tilde{B}_{s, x}-\frac{i \xi \eta}{\omega \varepsilon_{s} \mu_{s}} \tilde{B}_{s, y}\right. \\
& \left.+\frac{\eta}{\omega \varepsilon_{s}} n_{z} \delta\left(z-z_{d}\right)\right) \Delta z, \\
\frac{\Delta \tilde{B}_{s, x}}{\mu_{s}}= & \left(-\frac{i \xi \eta}{\omega \mu_{s}} \tilde{E}_{s, x}+i\left(\frac{\xi^{2}}{\omega \mu_{s}}-\omega \varepsilon_{s}\right) \tilde{E}_{s, y}\right. \\
& \left.+n_{y} \delta\left(z-z_{d}\right)\right) \Delta z, \\
\frac{\Delta \tilde{B}_{s, y}}{\mu_{s}}= & \left(i\left(\omega \varepsilon_{s}-\frac{\eta^{2}}{\omega \mu_{s}}\right) \tilde{E}_{s, x}+\frac{i \xi \eta}{\omega \mu_{s}} \tilde{E}_{s, y}\right. \\
& \left.-n_{x} \delta\left(z-z_{d}\right)\right) \Delta z .
\end{aligned}\right.
$$

Тогда в пределе $\Delta z \rightarrow 0$ скачок тангенциальных компонент напряженностей электрического и магнитного 
полей при переходе через бесконечно тонкий слой с током равен

$$
\left\{\begin{array}{l}
\Delta \tilde{E}_{s, x} \underset{\Delta z \rightarrow 0}{\longrightarrow} n_{z} \xi / \omega \varepsilon_{s}, \\
\Delta \tilde{E}_{s, y} \underset{\Delta z \rightarrow 0}{\longrightarrow} n_{z} \eta / \omega \varepsilon_{s}, \\
\Delta \tilde{B}_{s, x} / \mu_{s} \underset{\Delta z \rightarrow 0}{\longrightarrow} n_{y}, \\
\Delta \tilde{B}_{s, y} / \mu_{s} \underset{\Delta z \rightarrow 0}{\longrightarrow}-n_{x} .
\end{array}\right.
$$

В матричном виде уравнения, связывающие тангенциальные компоненты полей с двух сторон бесконечно тонкого слоя с током, можно записать в следующем виде:

$$
\left.\left(\begin{array}{c}
\tilde{E}_{s, x} \\
\tilde{E}_{s, y} \\
\tilde{B}_{s, x} / \mu_{s} \\
\tilde{B}_{s, y} / \mu_{s}
\end{array}\right)\right|_{z=z_{d}+0}-\left.\left(\begin{array}{c}
\tilde{E}_{s, x} \\
\tilde{E}_{s, y} \\
\tilde{B}_{s, x} / \mu_{s} \\
\tilde{B}_{s, y} / \mu_{s}
\end{array}\right)\right|_{z=z_{d}-0}=\left(\begin{array}{c}
n_{z} \xi / \omega \varepsilon_{s} \\
n_{z} \eta / \omega \varepsilon_{s} \\
n_{y} \\
-n_{x}
\end{array}\right) .
$$

Особо отметим, что скачок электрических полей при переходе через рассматриваемый бесконечно тонкий слой (при $\Delta z \rightarrow 0)$, вообще говоря зависит от диэлектрической проницаемости $\varepsilon_{s}$ среды, в которой расположен рассматриваемый источник тока. Но излучение источников, у которых есть только составляющие тока вдоль границы, не зависит в явном виде от диэлектрических свойств среды, в которой они расположены.

Выразим теперь левую часть граничного условия (31) через векторы-столбцы $\hat{\mathfrak{E}}_{1}$ и $\hat{\mathfrak{E}}_{N+1}$ полупространств снаружи плоскослоистой структуры. Для этого разобьем область с номером $s$ на две области и обозначим их индексами $l$ и $r$ (левая и правая, если смотреть на рис. 4). Введем вектор-столбцы $\hat{\mathfrak{E}}_{l}$ и $\hat{\mathfrak{E}}_{r}$ в этих областях. Тогда тангенциальные компоненты напряженностей полей в (31) можно выразить как

$$
\begin{aligned}
& \left.\left(\begin{array}{c}
\tilde{E}_{s, x} \\
\tilde{E}_{s, y} \\
\tilde{B}_{s, x} / \mu_{s} \\
\tilde{B}_{s, y} / \mu_{s}
\end{array}\right)\right|_{z=z_{d}-0}=\left(\begin{array}{cc}
e^{i \gamma_{s}\left(z_{d}-z_{s-1}\right)} \mathbf{I} & \mathbf{I} \\
e^{i \gamma_{s}\left(z_{d}-z_{s-1}\right)} \mathbf{G}_{s} & -\mathbf{G}_{s}
\end{array}\right) \hat{\mathfrak{E}}_{l}, \\
& \left.\left(\begin{array}{c}
\tilde{E}_{s, x} \\
\tilde{E}_{s, y} \\
\tilde{B}_{s, x} / \mu_{s} \\
\tilde{B}_{s, y} / \mu_{s}
\end{array}\right)\right|_{z=z_{d}+0}=\left(\begin{array}{cc}
\mathbf{I} & e^{i \gamma_{s}\left(z_{s}-z_{d}\right)} \mathbf{I} \\
\mathbf{G}_{s} & -e^{i \gamma_{s}\left(z_{s}-z_{d}\right)} \mathbf{G}_{s}
\end{array}\right) \hat{\mathfrak{E}}_{r} .
\end{aligned}
$$

Кроме того, из (28) следует, что

$$
\hat{\mathfrak{E}}_{1}=\mathbf{Q}_{L} \hat{\mathfrak{E}}_{l},
$$

где

$$
\begin{aligned}
& \mathbf{Q}_{L}=\left(\begin{array}{cc}
\mathbf{I} & \mathbf{I} \\
\mathbf{G}_{1} & -\mathbf{G}_{1}
\end{array}\right)^{-1} \times\left(\begin{array}{cc}
\mathbf{I} & e^{i \gamma_{2} d_{1} \mathbf{I}} \\
\mathbf{G}_{2} & -e^{i \gamma_{2} d_{1}} \mathbf{G}_{2}
\end{array}\right) \\
& \times\left(\begin{array}{cc}
e^{i \gamma_{2} d_{1} \mathbf{I}} & \mathbf{I} \\
e^{i \gamma_{2} d_{1}} \mathbf{G}_{2} & -\mathbf{G}_{2}
\end{array}\right)^{-1} \times \ldots \times\left(\begin{array}{cc}
\mathbf{I} & e^{i \gamma_{s-1} d_{s-2} \mathbf{I}} \\
\mathbf{G}_{s-1} & -e^{i \gamma_{s-1} d_{s-2} \mathbf{G}_{s-1}}
\end{array}\right) \\
& \times\left(\begin{array}{cc}
e^{i \gamma_{s-1} d_{s-2} \mathbf{I}} & \mathbf{I} \\
e^{i \gamma_{s-1} d_{s-2}} \mathbf{G}_{s-1} & -\mathbf{G}_{s-1}
\end{array}\right)^{-1} \times\left(\begin{array}{cc}
\mathbf{I} & e^{i \gamma_{s}\left(z_{d}-z_{s-1}\right)} \mathbf{I} \\
\mathbf{G}_{s} & -e^{i \gamma_{s}\left(z_{d}-z_{s-1}\right)} \mathbf{G}_{s}
\end{array}\right),
\end{aligned}
$$

а также

$$
\hat{\mathfrak{E}}_{r}=\mathbf{Q}_{R} \hat{\mathfrak{E}}_{N+1},
$$

где

$$
\begin{aligned}
& \mathbf{Q}_{R}=\left(\begin{array}{cc}
e^{i \gamma_{s}\left(z_{s}-z_{d}\right)} \mathbf{I} & \mathbf{I} \\
e^{i \gamma_{s}\left(z_{s}-z_{d}\right)} \mathbf{G}_{s} & -\mathbf{G}_{s}
\end{array}\right)^{-1} \times\left(\begin{array}{cc}
\mathbf{I} & e^{i \gamma_{s+1} d_{s} \mathbf{I}} \\
\mathbf{G}_{s+1} & -e^{i \gamma_{s+1} d_{s}} \mathbf{G}_{s+1}
\end{array}\right) \\
& \times\left(\begin{array}{cc}
e^{i \gamma_{s+1} d_{s} \mathbf{I}} & \mathbf{I} \\
e^{i \gamma_{s+1} d_{s}} \mathbf{G}_{s+1} & -\mathbf{G}_{s+1}
\end{array}\right)^{-1} \times \ldots \times\left(\begin{array}{cc}
\mathbf{I} & e^{i \gamma_{N} d_{N-1} \mathbf{I}} \\
\mathbf{G}_{N} & -e^{i \gamma_{N} d_{N-1}} \mathbf{G}_{N}
\end{array}\right) \\
& \times\left(\begin{array}{cc}
e^{i \gamma_{N} d_{N-1} \mathbf{I}} & \mathbf{I} \\
e^{i \gamma_{N} d_{N-1}} \mathbf{G}_{N} & -\mathbf{G}_{N}
\end{array}\right)^{-1} \times\left(\begin{array}{cc}
\mathbf{I} & \mathbf{I} \\
\mathbf{G}_{N+1} & -\mathbf{G}_{N+1}
\end{array}\right)
\end{aligned}
$$

Подставляя (34), (35) в (32), (33) и затем полученные выражения в (31), получаем

$$
\mathbf{H}_{R} \times \hat{\mathfrak{E}}_{N+1}=\mathbf{H}_{L} \times \hat{\mathfrak{E}}_{1}+\mathbf{V},
$$

где $\mathbf{V}=\left(n_{z} \xi / \omega \varepsilon_{s} ; n_{z} \eta / \omega \varepsilon_{s} ; n_{y} ;-n_{x}\right)^{T}$ - вектор-столбец, характеризующий возбуждающее воздействие на систему стороннего элементарного тока, а матрицы $\mathbf{H}_{R}$ и $\mathbf{H}_{L}$ - характеризуют отклик на внешнее возбуждение слоистой структуры справа и слева излучателя и выражаются следующим образом:

$$
\begin{aligned}
\mathbf{H}_{R} & =\left(\begin{array}{cc}
\mathbf{I} & e^{i \gamma_{s}\left(z_{s}-z_{d}\right)} \mathbf{I} \\
\mathbf{G}_{s} & -e^{i \gamma_{s}\left(z_{s}-z_{d}\right)} \mathbf{G}_{s}
\end{array}\right) \mathbf{Q}_{R} \\
& =\mathbf{T}_{R} \times\left(\prod_{m=s+1}^{N} \mathbf{T}_{m}\right) \times \mathbf{T}_{N+1}, \\
\mathbf{H}_{L} & =\left(\begin{array}{cc}
e^{i \gamma_{s}\left(z_{d}-z_{s-1}\right)} \mathbf{I} & \mathbf{I} \\
e^{i \gamma_{s}\left(z_{d}-z_{s-1}\right)} \mathbf{G}_{s} & -\mathbf{G}_{s}
\end{array}\right)\left(\mathbf{Q}_{L}\right)^{-1} \\
& =\left(\mathbf{T}_{1} \times\left(\prod_{m=2}^{s-1} \mathbf{T}_{m}\right) \times \mathbf{T}_{L}\right)^{-1},
\end{aligned}
$$

где матрицы $\mathbf{T}_{m}$ при $m \neq s$ выражаются формулой

$$
\mathbf{T}_{m}=\left(\begin{array}{cc}
\mathbf{I} & e^{i \gamma_{m} d_{m-1} \mathbf{I}} \\
\mathbf{G}_{m} & -e^{i \gamma_{m} d_{m-1}} \mathbf{G}_{m}
\end{array}\right)\left(\begin{array}{cc}
e^{i \gamma_{m} d_{m-1} \mathbf{I}} & \mathbf{I} \\
e^{i \gamma_{m} d_{m-1}} \mathbf{G}_{m} & -\mathbf{G}_{m}
\end{array}\right)^{-1},
$$

а матрицы $\mathbf{T}_{L}$ и $\mathbf{T}_{R}-$ формулами

$$
\mathbf{T}_{L}=\left(\begin{array}{cc}
\mathbf{I} & e^{i \gamma_{s}\left(z_{d}-z_{s-1}\right)} \mathbf{I} \\
\mathbf{G}_{s} & -e^{i \gamma_{s}\left(z_{d}-z_{s-1}\right)} \mathbf{G}_{s}
\end{array}\right)\left(\begin{array}{cc}
e^{i \gamma_{s}\left(z_{d}-z_{s-1}\right)} \mathbf{I} & \mathbf{I} \\
e^{i \gamma_{s}\left(z_{d}-z_{s-1}\right)} \mathbf{G}_{s} & -\mathbf{G}_{s}
\end{array}\right)^{-1},
$$




$$
\mathbf{T}_{R}=\left(\begin{array}{cc}
\mathbf{I} & e^{i \gamma_{s}\left(z_{s}-z_{d}\right)} \mathbf{I} \\
\mathbf{G}_{s} & -e^{i \gamma_{s}\left(z_{s}-z_{d}\right)} \mathbf{G}_{s}
\end{array}\right)\left(\begin{array}{cc}
e^{i \gamma_{s}\left(z_{s}-z_{d}\right)} \mathbf{I} & \mathbf{I} \\
e^{i \gamma_{s}\left(z_{s}-z_{d}\right)} \mathbf{G}_{s} & -\mathbf{G}_{s}
\end{array}\right)^{-1} .
$$

В вышеприведенных формулах матрицы $\mathbf{G}_{s}$ выражаются по формулам (16).

Далее, в рассматриваемой задаче источники полей находятся исключительно внутри плоскослоистой структуры. Поэтому в столбцах $\mathfrak{\mathfrak { E }}_{1}$ и $\hat{\mathfrak{E}}_{N+1}$ есть только компоненты волн, идущие от плоскослоистой структуры. Поэтому эти столбцы имеют вид

$$
\begin{gathered}
\hat{\mathfrak{E}}_{1}=\left(0 ; 0 ; \hat{E}_{1, x}^{-} ; \hat{E}_{1, y}^{-}\right)^{T}, \\
\hat{\mathfrak{E}}_{N+1}=\left(\hat{E}_{N+1, x}^{+} ; \hat{E}_{N+1, y}^{+} ; 0 ; 0\right)^{T} .
\end{gathered}
$$

Чтобы получить оставшиеся, отличные от нуля, компоненты $\hat{\mathfrak{E}}_{1}$ и $\hat{\mathfrak{E}}_{N+1}$, разобьем матрицы $\mathbf{H}_{R}$ и $\mathbf{H}_{L}$ каждую на четыре $2 \times 2$ подматрицы $\mathbf{H}_{R A}, \mathbf{H}_{R B}, \mathbf{H}_{R C}, \mathbf{H}_{R D}$ и $\mathbf{H}_{L A}$, $\mathbf{H}_{L B}, \mathbf{H}_{L C}, \mathbf{H}_{L D}$ следующим образом:

$$
\mathbf{H}_{R}=\left(\begin{array}{ll}
\mathbf{H}_{R A} & \mathbf{H}_{R B} \\
\mathbf{H}_{R C} & \mathbf{H}_{R D}
\end{array}\right) \text { и } \quad \mathbf{H}_{L}=\left(\begin{array}{ll}
\mathbf{H}_{L A} & \mathbf{H}_{L B} \\
\mathbf{H}_{L C} & \mathbf{H}_{L D}
\end{array}\right),
$$

тогда уравнение (36) примет вид

$$
\left(\begin{array}{cc}
\mathbf{H}_{R A} & \mathbf{H}_{R B} \\
\mathbf{H}_{R C} & \mathbf{H}_{R D}
\end{array}\right) \times\left(\begin{array}{c}
\hat{E}_{N+1, x}^{+} \\
\hat{E}_{N+1, y}^{+} \\
0 \\
0
\end{array}\right)=\left(\begin{array}{cc}
\mathbf{H}_{L A} & \mathbf{H}_{L B} \\
\mathbf{H}_{L C} & \mathbf{H}_{L D}
\end{array}\right) \times\left(\begin{array}{c}
0 \\
0 \\
\hat{E}_{1, x}^{-} \\
\hat{E}_{1, y}^{-}
\end{array}\right)+\mathbf{V} .
$$

Если еще разбить вектор $\mathbf{V}=\left(V_{1} ; V_{2} ; V_{3} ; V_{4}\right)^{T}$ на $\mathbf{V}_{A}=\left(V_{1}, V_{2}\right)^{T}$ и $\mathbf{V}_{B}=\left(V_{3}, V_{4}\right)^{T}$, то уравнение (37) можно представить следующей системой из двух матричных уравнений:

$$
\begin{aligned}
\mathbf{H}_{R A}\left(\begin{array}{c}
\hat{E}_{N+1, x}^{+} \\
\hat{E}_{N+1, y}^{+}
\end{array}\right) & =\mathbf{H}_{L B}\left(\begin{array}{c}
\hat{E}_{1, x}^{-} \\
\hat{E}_{1, y}^{-}
\end{array}\right)+\mathbf{V}_{A}, \\
\mathbf{H}_{R C}\left(\begin{array}{c}
\hat{E}_{N+1, x}^{+} \\
\hat{E}_{N+1, y}^{+}
\end{array}\right) & =\mathbf{H}_{L D}\left(\begin{array}{c}
\hat{E}_{1, x}^{-} \\
\hat{E}_{1, y}^{-}
\end{array}\right)+\mathbf{V}_{B} .
\end{aligned}
$$

Полученные уравнения можно снова объединить в одно матричное $4 \times 4$ уравнение:

$$
\left(\begin{array}{ll}
-\mathbf{H}_{L B} & \mathbf{H}_{R A} \\
-\mathbf{H}_{L D} & \mathbf{H}_{R C}
\end{array}\right) \hat{\mathfrak{E}}_{\mathrm{out}}=\mathbf{V},
$$

где введен вектор-столбец

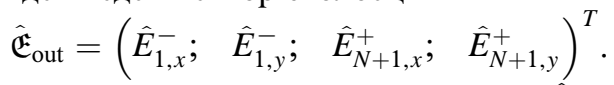

Решая это уравнение, найдем $\hat{E}_{1, x}^{-}, \hat{E}_{1, y}^{-}$и $\hat{E}_{N+1, x}^{+}$, $\hat{E}_{N+1, y}^{+}$, а значит, уходящую из плоскослоистой структуры влево волну (в направлении $z \rightarrow-\infty$ ) по формулам

$$
\begin{aligned}
\mathbf{E}_{1}(x, y, z)= & \frac{1}{(2 \pi)^{2}} \int_{-\infty}^{+\infty} \int_{-\infty}^{+\infty}\left(\begin{array}{cc}
1 & 0 \\
0 & 1 \\
\xi / \gamma_{1} & \eta / \gamma_{1}
\end{array}\right)\left(\begin{array}{c}
\hat{E}_{1, x}^{-} \\
\hat{E}_{1, y}^{-}
\end{array}\right) \\
& \times e^{-i \gamma_{1} z} e^{i(\xi x+\eta y)} d \xi d \eta,
\end{aligned}
$$

$$
\begin{aligned}
& \mathbf{B}_{1}(x, y, z)= \\
& =\frac{1}{(2 \pi)^{2}} \int_{-\infty}^{+\infty} \int_{-\infty}^{+\infty}\left(\begin{array}{cc}
\xi \eta / \omega \gamma_{1} & \left(\gamma_{1}^{2}+\eta^{2}\right) / \omega \gamma_{1} \\
-\left(\gamma_{1}^{2}+\xi^{2}\right) / \omega \gamma_{1} & -\xi \eta / \omega \gamma_{1} \\
-\eta / \omega & \xi / \omega
\end{array}\right) \\
& \times\left(\begin{array}{l}
\hat{E}_{1, x}^{-} \\
\hat{E}_{1, y}^{-}
\end{array}\right) e^{-i \gamma_{1} z} e^{i(\xi x+\eta y)} d \xi d \eta
\end{aligned}
$$

и волну вправо от плоскослоистой структуры (в направлении $z \rightarrow+\infty)$ по формулам

$$
\begin{aligned}
\mathbf{E}_{N+1}(x, y, z)=\frac{1}{(2 \pi)^{2}} \int_{-\infty}^{+\infty} \int_{-\infty}^{+\infty}\left(\begin{array}{cc}
1 & 0 \\
0 & 1 \\
-\xi / \gamma_{N+1} & -\eta / \gamma_{N+1}
\end{array}\right) \\
\times\left(\begin{array}{c}
\hat{E}_{N+1, x}^{+} \\
\hat{E}_{N+1, y}^{+}
\end{array}\right) e^{i \gamma_{N+1}\left(z-z_{N}\right)} e^{i(\xi x+\eta y)} d \xi d \eta,
\end{aligned}
$$

$\mathbf{B}_{N+1}(x, y, z)=$

$=\frac{1}{(2 \pi)^{2}} \int_{-\infty-\infty}^{+\infty} \int_{-\infty}^{-\xi \eta / \omega \gamma_{N+1}}\left(\begin{array}{cc}-\left(\gamma_{N+1}^{2}+\eta^{2}\right) / \omega \gamma_{N+1} \\ \left(\gamma_{N+1}^{2}+\xi^{2}\right) / \omega \gamma_{N+1} & \xi \eta / \omega \gamma_{N+1} \\ -\eta / \omega & \xi / \omega\end{array}\right)$

$\times\left(\begin{array}{c}\hat{E}_{N+1, x}^{+} \\ \hat{E}_{N+1, y}^{+}\end{array}\right) e^{i \gamma_{N+1}\left(z-z_{N}\right)} e^{i(\xi x+\eta y)} d \xi d \eta$.

Наконец, при необходимости, зная $\hat{\mathfrak{E}}_{1}$ и $\hat{\mathfrak{E}}_{N+1}$, можно найти векторы-столбцы поля в любой внутренней области $\hat{\mathfrak{E}}_{j}$, так как они однозначно определяется граничными условиями. После этого электромагнитные поля в любой из этих областей могут быть найдены по формулам (10) и (13). Таким образом, электромагнитные поля будут определены во всем пространстве.

\section{Поля в полупространствах}

Заметим, что из формул (39), (41) мы можем найти компоненты тангенциального электрического поля на границах полупространств по следующим формулам: на границе $z=z_{0}=0$

$$
\left\{\begin{array}{l}
E_{1, x}(x, y, 0)=\frac{1}{(2 \pi)^{2}} \int_{-\infty}^{+\infty} \int_{-\infty}^{+\infty} \hat{E}_{1, x}^{-} e^{i(\xi x+\eta y)} d \xi d \eta, \\
E_{1, y}(x, y, 0)=\frac{1}{(2 \pi)^{2}} \int_{-\infty}^{+\infty} \int_{-\infty}^{+\infty} \hat{E}_{1, y}^{-} e^{i(\xi x+\eta y)} d \xi d \eta,
\end{array}\right.
$$

на границе $z=z_{N}=d_{\text {tot }}$

$$
\left\{\begin{array}{l}
E_{N+1, x}\left(x, y, z_{N}\right)=\frac{1}{(2 \pi)^{2}} \int_{-\infty}^{+\infty} \int_{-\infty}^{+\infty} \hat{E}_{N+1, x}^{+} e^{i(\xi x+\eta y)} d \xi d \eta, \\
E_{N+1, y}\left(x, y, z_{N}\right)=\frac{1}{(2 \pi)^{2}} \int_{-\infty}^{+\infty} \int_{-\infty}^{+\infty} \hat{E}_{N+1, y}^{+} e^{i(\xi x+\eta y)} d \xi d \eta .
\end{array}\right.
$$


Тогда в полупространствах мы можем воспользоваться принципом эквивалентности и воспользоваться формулами Стрэттона и Чу [21,22] в формулировке для полуплоскости [23,24], согласно которой электромагнитные поля в полупространствах могут быть найдены только по распределению тангенциального электрического поля на границе по формулам

$$
\begin{gathered}
\mathbf{E}(\mathbf{r})=-\operatorname{rot} \mathbf{A}_{m}(\mathbf{r}), \\
\mathbf{H}(\mathbf{r})=i \omega \varepsilon_{j} \mathbf{A}_{m}(\mathbf{r})-\frac{1}{i \omega \mu_{j}} \operatorname{graddiv} \mathbf{A}_{m}(\mathbf{r}),
\end{gathered}
$$

где

$$
\mathbf{A}_{m}(\mathbf{r})=\frac{1}{2 \pi} \int_{P}[\mathbf{E}, \mathbf{n}] \frac{e^{i k_{j}\left|\mathbf{r}-\mathbf{r}_{p}\right|}}{\left|\mathbf{r}-\mathbf{r}_{p}\right|} d s_{p},
$$

n - внутренняя нормаль рассматриваемого полупространства, $\quad \mathbf{r}=x \mathbf{e}_{x}+y \mathbf{e}_{y}+z \mathbf{e}_{z}$ радиус-вектор точки наблюдения, в которой находятся поля, $\quad \mathbf{r}_{p}=x_{p} \mathbf{e}_{x}+y_{p} \mathbf{e}_{y}+z_{p} \mathbf{e}_{z}$ радиус-вектор точки интегрирования, $\left|\mathbf{r}-\mathbf{r}_{p}\right|=\sqrt{\left(x-x_{p}\right)^{2}+\left(y-y_{p}\right)^{2}+\left(z-z_{p}\right)^{2}}$ расстояние между точками $\mathbf{r}$ и $\mathbf{r}_{p}$, причем дифференцирование в операторах производится по координатам точки наблюдения, а интегрирование идет по всей плоской границе $P$ пленки.

Замечая, что $[\mathbf{E}, \mathbf{n}]=\mathbf{E}_{\tau}-$ тангенциальная составляющая поля на границе, в полупространстве $N+1$ получим

$$
\begin{aligned}
& {\left[\mathbf{E}_{\tau}, \mathbf{n}\right]=\left|\begin{array}{ccc}
\mathbf{e}_{x} & \mathbf{e}_{y} & \mathbf{e}_{z} \\
E_{N+1, x} & E_{N+1, y} & 0 \\
0 & 0 & 1
\end{array}\right|=E_{N+1, y} \mathbf{e}_{x}-E_{N+1, x} \mathbf{e}_{y},} \\
& \mathbf{A}_{m}(\mathbf{r})=\mathbf{e}_{x}\left(\frac{1}{2 \pi} \int_{-\infty}^{+\infty} \int_{-\infty}^{+\infty} E_{N+1, y}\left(x_{p}, y_{p}, z_{N}\right)\right. \\
& \left.\times \frac{e^{i k_{N+1} \sqrt{\left(x-x_{p}\right)^{2}+\left(y-y_{p}\right)^{2}+(z-d)^{2}}}}{\sqrt{\left(x-x_{p}\right)^{2}+\left(y-y_{p}\right)^{2}+\left(z-z_{N}\right)^{2}}} d x_{p} d y_{p}\right)- \\
& -\mathbf{e}_{y}\left(\frac{1}{2 \pi} \int_{-\infty}^{+\infty} \int_{-\infty}^{+\infty} E_{N+1, x}\left(x_{p}, y_{p}, z_{N}\right)\right. \\
& \left.\quad \times \frac{e^{i k_{N+1} \sqrt{\left(x-x_{p}\right)^{2}+\left(y-y_{p}\right)^{2}+(z-d)^{2}}}}{\sqrt{\left(x-x_{p}\right)^{2}+\left(y-y_{p}\right)^{2}+\left(z-z_{N}\right)^{2}}} d x_{p} d y_{p}\right)
\end{aligned}
$$

или

$$
\begin{aligned}
& \mathbf{A}_{m}(\mathbf{r})= \\
& =\mathbf{e}_{x}\left(\frac{1}{2 \pi} \int_{-\infty}^{+\infty} \int_{-\infty}^{+\infty} E_{N+1, y}\left(x_{p}, y_{p}, z_{N}\right) \frac{e^{i k_{N+1}\left|\mathbf{r}-\mathbf{r}_{p}\right|}}{\left|\mathbf{r}-\mathbf{r}_{p}\right|} d x_{p} d y_{p}\right) \\
& -\mathbf{e}_{y}\left(\frac{1}{2 \pi} \int_{-\infty}^{+\infty} \int_{-\infty}^{+\infty} E_{N+1, x}\left(x_{p}, y_{p}, z_{N}\right) \frac{e^{i k_{N+1}\left|\mathbf{r}-\mathbf{r}_{p}\right|}}{\left|\mathbf{r}-\mathbf{r}_{p}\right|} d x_{p} d y_{p}\right) .
\end{aligned}
$$

Тогда по формуле $\mathbf{E}(\mathbf{r})=-\operatorname{rot} \mathbf{A}_{m}(\mathbf{r})$ составляющие электрического поля определятся следующими выражениями

$$
\begin{aligned}
& E_{N+1, x}(x, y, z)=\frac{1}{2 \pi} \int_{-\infty}^{+\infty} \int_{-\infty}^{+\infty} E_{N+1, x}\left(x_{p}, y_{p}, z_{N}\right) \\
& \times \frac{\left(z-z_{p}\right)\left(1-i k_{N+1}\left|\mathbf{r}-\mathbf{r}_{p}\right|\right)}{\left|\mathbf{r}-\mathbf{r}_{p}\right|^{3}} e^{i k_{N+1}\left|\mathbf{r}-\mathbf{r}_{p}\right|} d x_{p} d y_{p}, \\
& E_{N+1, y}(x, y, z)=\frac{1}{2 \pi} \int_{-\infty}^{+\infty} \int_{-\infty}^{+\infty} E_{N+1, y}\left(x_{p}, y_{p}, z_{N}\right) \\
& \times \frac{\left(z-z_{p}\right)\left(1-i k_{N+1}\left|\mathbf{r}-\mathbf{r}_{p}\right|\right)}{\left|\mathbf{r}-\mathbf{r}_{p}\right|^{3}} e^{i k_{N+1}\left|\mathbf{r}-\mathbf{r}_{p}\right|} d x_{p} d y_{p},
\end{aligned}
$$

$E_{N+1, z}(x, y, z)=$

$$
\begin{aligned}
& =-\frac{1}{2 \pi} \int_{-\infty}^{+\infty} \int_{-\infty}^{+\infty}\left(\left(x-x_{p}\right) E_{N+1, x}\left(x_{p}, y_{p}, z_{N}\right)+\left(y-y_{p}\right)\right. \\
& \left.\times E_{N+1, y}\left(x_{p}, y_{p}, z_{N}\right)\right) \frac{\left(1-i k_{N+1}\left|\mathbf{r}-\mathbf{r}_{p}\right|\right) e^{i k_{N+1}\left|\mathbf{r}-\mathbf{r}_{p}\right|}}{\left|\mathbf{r}-\mathbf{r}_{p}\right|^{3}} d x_{p} d y_{p} .
\end{aligned}
$$

В принципе выражения (45)-(47) позволяют точно определить электрическое поле в любой точке полупространства, если подставить в них выражения (44), однако с вычислительной точки зрения четырехкратное интегрирование не является привлекательным. Значительное упрощение получается при рассмотрении полей в дальней зоне полученной излучающей плоской апертуры. Действительно, рассмотрим поля в дальней зоне

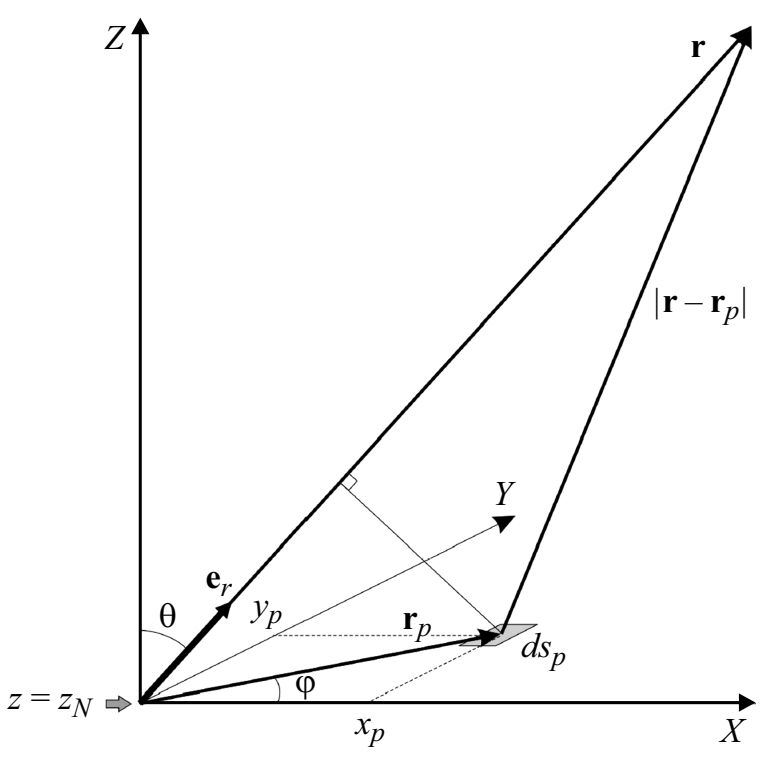

Рис. 5. Геометрия задачи нахождения электрического поля излучения в дальней зоне при интегрировании по формулам $(45)-(47)$. 
источника $|\mathbf{r}| \gg\left|\mathbf{r}_{p}\right|$, причем $\mathbf{r}=x \mathbf{e}_{x}+y \mathbf{e}_{y}+\left(z-z_{N}\right) \mathbf{e}_{z}$ (рис. 5) в полупространстве $N+1$.

Будем считать, что тангенциальное электрическое поле сосредоточено в основном в вблизи источника. Тогда в подынтегральном выражении в (45) получим в пределе дальней зоны, где $\mathbf{r}$ и $\mathbf{r}_{p}$ практически параллельны, выражения

$$
\begin{aligned}
& \frac{\left(z-z_{N}\right)\left(1-i k_{N+1}\left|\mathbf{r}-\mathbf{r}_{p}\right|\right)}{\left|\mathbf{r}-\mathbf{r}_{p}\right|^{3}} e^{i k_{N+1}\left|\mathbf{r}-\mathbf{r}_{p}\right|} \rightarrow \\
& \rightarrow-i k_{N+1} \frac{\left(z-z_{N}\right)}{|\mathbf{r}|} \frac{e^{i k_{N+1}|\mathbf{r}|}}{|\mathbf{r}|} e^{i\left(\mathbf{k}_{N+1}, \mathbf{r}_{p}\right)} \\
& =-i k_{N+1} \cos \theta \frac{e^{i k_{N+1}|\mathbf{r}|}}{|\mathbf{r}|} e^{i\left(\mathbf{k}_{N+1}, \mathbf{r}_{p}\right)},
\end{aligned}
$$

где $\theta-$ полярный угол, а вектор $\mathbf{k}_{N+1}$ равен по модулю $k_{N+1}=\omega \sqrt{\varepsilon_{N+1} \mu_{N+1}}$ и направлен вдоль единичного вектора $\mathbf{e}_{r}=\mathbf{r} /|\mathbf{r}|$. Тогда получим в дальней зоне

$$
\begin{aligned}
& E_{N+1, x}(x, y, z) \approx-\frac{i k_{N+1} \cos \theta e^{i k_{N+1}|\mathbf{r}|}}{2 \pi|\mathbf{r}|} \\
& \times \int_{-\infty}^{+\infty} \int_{-\infty}^{+\infty} E_{N+1, x}\left(x_{p}, y_{p}, z_{N}\right) e^{-i\left(\mathbf{k}_{N+1}, \mathbf{r}_{p}\right)} d x_{p} d y_{p} .
\end{aligned}
$$

Рассмотрим подробнее двойной интеграл в (48):

$$
\begin{aligned}
& \int_{-\infty}^{+\infty} \int_{-\infty}^{+\infty} E_{N+1, x}\left(x_{p}, y_{p}, z_{N}\right) e^{-i\left(\mathbf{k}_{N+1}, \mathbf{r}_{p}\right)} d x_{p} d y_{p}= \\
& =\int_{-\infty}^{+\infty} \int_{-\infty}^{+\infty} E_{N+1, x}\left(x_{p}, y_{p}, z_{N}\right) \\
& \quad \times e^{-i\left(x_{p} k_{N+1} \sin \theta \cos \varphi+y_{p} k_{N+1} \sin \theta \sin \varphi\right)} d x_{p} d y_{p},
\end{aligned}
$$

где $\varphi-$ угол в плоскости $X Y$ сферической системы координат (рис. 5).

Подставляя выражение (44), получим

$$
\begin{aligned}
& \int_{-\infty}^{+\infty} \int_{-\infty}^{+\infty} E_{N+1, x}\left(x_{p}, y_{p}, z_{N}\right) e^{-i\left(\mathbf{k}_{N+1}, \mathbf{r}_{p}\right)} d x_{p} d y_{p}= \\
& =\frac{1}{(2 \pi)^{2}} \int_{-\infty}^{+\infty} \int_{-\infty}^{+\infty}\left(\int_{-\infty}^{+\infty} \int_{-\infty}^{+\infty} \hat{E}_{N+1, x}^{+}(\xi, \eta) e^{i\left(\xi x_{p}+\eta y_{p}\right)} d \xi d \eta\right) \\
& \times e^{-i\left(x_{p} k_{N+1} \sin \theta \cos \varphi+y_{p} k_{N+1} \sin \theta \sin \varphi\right)} d x_{p} d y_{p}= \\
& =\frac{1}{(2 \pi)^{2}} \int_{-\infty}^{+\infty} \int_{-\infty}^{+\infty} \hat{E}_{N+1, x}^{+}(\xi, \eta)
\end{aligned}
$$$$
\times\left(\int_{-\infty}^{+\infty} \int_{-\infty}^{+\infty} e^{i\left(\left(\xi-k_{N+1} \sin \theta \cos \varphi\right) x_{p}+\left(\eta-k_{N+1} \sin \theta \sin \varphi\right) y_{p}\right)} d x_{p} d y_{p}\right) d \xi d \eta
$$

Учитывая известное свойство $\delta$-функции

$$
\begin{aligned}
& \int_{-\infty}^{+\infty} \int_{-\infty}^{+\infty} e^{i\left(\left(\xi-k_{N+1} \sin \theta \cos \varphi\right) x_{p}+\left(\eta-k_{N+1} \sin \theta \sin \varphi\right) y_{p}\right)} d x_{p} d y_{p} \\
& =(2 \pi)^{2} \delta\left(\xi-k_{N+1} \sin \theta \cos \varphi\right) \delta\left(\eta-k_{N+1} \sin \theta \sin \varphi\right),
\end{aligned}
$$

получим

$$
\begin{aligned}
& \int_{-\infty}^{+\infty} \int_{-\infty}^{+\infty} E_{N+1, x}\left(x_{p}, y_{p}, z_{N}\right) e^{-i\left(\mathbf{k}_{N+1}, \mathbf{r}_{p}\right)} d x_{p} d y_{p} \\
& =\hat{E}_{N+1, x}^{+}\left(k_{N+1} \sin \theta \cos \varphi, k_{N+1} \sin \theta \sin \varphi\right)
\end{aligned}
$$

где $\hat{E}_{N+1, x}^{+}(\xi, \eta)$ - известная из решения уравнения (38) функция.

Тогда для элементарного диполя составляющая $E_{N+1, x}$ электрического поля в рассматриваемом полупространстве в дальней зоне вычисляется по формуле

$$
\begin{aligned}
& E_{N+1, x}(x, y, z) \approx-\frac{i k_{N+1} \cos \theta e^{i k_{N+1}|\mathbf{r}|}}{2 \pi|\mathbf{r}|} \\
& \times \hat{E}_{N+1, x}^{+}\left(k_{N+1} \sin \theta \cos \varphi, k_{N+1} \sin \theta \sin \varphi\right),
\end{aligned}
$$

где $E_{N+1, y}^{+}(\xi, \eta)$ - известная из решения уравнения (38) функция.

Аналогично для составляющей $E_{N+1, y}$ в дальней зоне получим формулу

$$
\begin{aligned}
& E_{N+1, y}(x, y, z) \approx-\frac{i k_{N+1} \cos \theta e^{i k_{N+1}|\mathbf{r}|}}{2 \pi|\mathbf{r}|} \\
& \times \hat{E}_{N+1, y}^{+}\left(k_{N+1} \sin \theta \cos \varphi, k_{N+1} \sin \theta \sin \varphi\right) .
\end{aligned}
$$

Наконец, для составляющей $E_{N+1, z}$ в дальней зоне получим формулу

$$
\begin{aligned}
& E_{N+1, z}(x, y, z) \approx \frac{i k_{N+1} \sin \theta e^{i k_{N+1}|\mathbf{r}|}}{2 \pi|\mathbf{r}|} \\
& \times\left(\cos \varphi \hat{E}_{N+1, x}^{+}\left(k_{N+1} \sin \theta \cos \varphi, k_{N+1} \sin \theta \sin \varphi\right)+\right. \\
& \left.+\sin \varphi \hat{E}_{N+1, y}^{+}\left(k_{N+1} \sin \theta \cos \varphi, k_{N+1} \sin \theta \sin \varphi\right)\right)
\end{aligned}
$$

Итак, мы нашли составляющие электрического поля в точке с координатами $x=r \sin \theta \cos \varphi, y=r \sin \theta \sin \varphi$, $z=r \cos \theta+z_{N}$.

Действуя аналогично, учитывая, что для полупространства $j=1$ внутренняя нормаль изменит знак, найдем электрическое поле в дальней зоне по следующим формулам:

$$
\begin{aligned}
& E_{1, x}(x, y, z) \approx \frac{i k_{1} \cos \theta e^{i k_{1}|\mathbf{r}|}}{2 \pi|\mathbf{r}|} \\
& \times \hat{E}_{1, x}^{-}\left(k_{1} \sin \theta \cos \varphi, k_{1} \sin \theta \sin \varphi\right),
\end{aligned}
$$




$$
\begin{gathered}
E_{1, y}(x, y, z) \approx \frac{i k_{1} \cos \theta e^{i k_{1}|\mathbf{r}|}}{2 \pi|\mathbf{r}|} \\
\times \hat{E}_{1, y}^{-}\left(k_{1} \sin \theta \cos \varphi, k_{1} \sin \theta \sin \varphi\right), \\
E_{1, z}(x, y, z) \approx-\frac{i k_{1} \sin \theta e^{i k_{1}|\mathbf{r}|}}{2 \pi|\mathbf{r}|} \\
\times\left(\cos \varphi \hat{E}_{1, x}^{-}\left(k_{1} \sin \theta \cos \varphi, k_{1} \sin \theta \sin \varphi\right)+\right. \\
\left.+\sin \varphi \hat{E}_{1, y}^{-}\left(k_{N+1} \sin \theta \cos \varphi, k_{1} \sin \theta \sin \varphi\right)\right),
\end{gathered}
$$

где координаты точки, в которой находится поле, вычисляют по формулам $x=r \sin \theta \cos \varphi, y=r \sin \theta \sin \varphi$, $z=r \cos \theta$, причем $\pi / 2 \leq \theta \leq \pi$ в области $j=1$, а функции $\hat{E}_{1, x}^{-}(\xi, \eta)$ и $\hat{E}_{1, y}^{-}(\xi, \eta)$ - решения уравнения $(38)$.

Итак, мы получили выражения для электрических полей, излученные элементарным диполем в многопленочной структуре в окружающие полупространства. Отметим, что полученные выражения не требуют интегрирования и требуют решения только матричного уравнения (38).

\section{Поля в дальней зоне от элементарных горизонтального и вертикального диполей, расположенных на границе металлической пленки, нанесенной на поверхность призмы}

Рассмотрим теперь задачу излучения элементарного горизонтального источника, расположенного на свободной границе пленки (рис. 6, $a$ ) в схеме Кречмана. Поляризация источника - вдоль оси $X\left(n_{x}=1, x_{y}=0\right.$, $\left.n_{z}=0\right)$. Толщина пленки равна $d$. Индекс 1 соответствует полупространству материала призмы, 2 - пленке, а 3 - свободному полупространству над пленкой. Тогда $N=2, z_{d}=z_{2}=d, \mathbf{H}_{R}=\mathbf{T}_{3}, \mathbf{H}_{L}=\left(\mathbf{T}_{1} \times \mathbf{T}_{2}\right)^{-1}$ и уравнение (36) примет вид

$$
\mathbf{T}_{3} \times \hat{\mathfrak{E}}_{3}=\left(\mathbf{T}_{1} \times \mathbf{T}_{2}\right)^{-1} \times \hat{\mathfrak{E}}_{1}+\mathbf{V},
$$

где матрицы выражаются следующими формулами:

$$
\begin{gathered}
\mathbf{T}_{1}=\left(\begin{array}{cc}
\mathbf{I} & \mathbf{I} \\
\mathbf{G}_{1} & -\mathbf{G}_{1}
\end{array}\right)^{-1}, \\
\mathbf{T}_{2}=\left(\begin{array}{cc}
\mathbf{I} & e^{i \gamma_{2} d} \mathbf{I} \\
\mathbf{G}_{2} & -e^{i \gamma_{2} d} \mathbf{G}_{2}
\end{array}\right)\left(\begin{array}{cc}
e^{i \gamma_{2} d} \mathbf{I} & \mathbf{I} \\
e^{i \gamma_{2} d} \mathbf{G}_{2} & -\mathbf{G}_{2}
\end{array}\right)^{-1}, \\
\mathbf{T}_{3}=\left(\begin{array}{cc}
\mathbf{I} & \mathbf{I} \\
\mathbf{G}_{3} & -\mathbf{G}_{3}
\end{array}\right),
\end{gathered}
$$

а вектор-столбец стороннего тока равен $\mathbf{V}=(0 ; 0 ; 0 ;-1)^{T}$.

Учитывая, что $\left(\mathbf{T}_{1} \times \mathbf{T}_{2}\right)^{-1}=\mathbf{T}_{2}^{-1} \times \mathbf{T}_{1}^{-1}$, получим

$\mathbf{H}_{L}=\left(\mathbf{T}_{1} \times \mathbf{T}_{2}\right)^{-1}=\mathbf{T}_{2}^{-1} \times \mathbf{T}_{1}^{-1}$

$=\left(\begin{array}{cc}e^{i \gamma_{2} d} \mathbf{I} & \mathbf{I} \\ e^{i \gamma_{2} d} \mathbf{G}_{2} & -\mathbf{G}_{2}\end{array}\right)\left(\begin{array}{cc}\mathbf{I} & e^{i \gamma_{2} d} \mathbf{I} \\ \mathbf{G}_{2} & -e^{i \gamma_{2} d} \mathbf{G}_{2}\end{array}\right)^{-1} \times\left(\begin{array}{cc}\mathbf{I} & \mathbf{I} \\ \mathbf{G}_{1} & -\mathbf{G}_{1}\end{array}\right)$.
Вводя вектор-столбец $\hat{\mathfrak{E}}_{\text {out }}=\left(\hat{E}_{1, x}^{-} ; \hat{E}_{1, y}^{-} ; \hat{E}_{3, x}^{+} ; \hat{E}_{3, y}^{+}\right)^{T}$, уравнение (38) для данной задачи примет вид

$$
\left(\begin{array}{cc}
-\mathbf{H}_{L B} & \mathbf{I} \\
-\mathbf{H}_{L D} & \mathbf{G}_{3}
\end{array}\right) \times \hat{\mathfrak{E}}_{\text {out }}=\mathbf{V}
$$

Решая линейное уравнение (56), найдем $\hat{E}_{1, x}^{-}, \hat{E}_{1, y}^{-}$и $\hat{E}_{3, x}^{+}, \hat{E}_{3, y}^{+}$, и далее по формулам (49)-(54) при $N=2$, $z_{d}=z_{2}=d$ получим компоненты электрического поля в дальней зоне горизонтального диполя в призме (в область 1) и свободном полупространстве (область 3) над пленкой.

Задача излучения элементарного вертикального источника, расположенного на свободной границе пленки в схеме Кречмана (рис. 6, $b$ ) решается аналогично. Будем считать, что источник находится в свободном полупространстве (в области 3) бесконечно близко к границе. Поляризация источника - вдоль оси $Z\left(n_{x}=0\right.$, $\left.n_{y}=0, n_{z}=1\right)$. Применяя те же рассуждения, что и для горизонтального диполя, получим для фурье-образов полей $\hat{E}_{1, x}^{-}, \hat{E}_{1, y}^{-}$и $\hat{E}_{3, x}^{+}, \hat{E}_{3, y}^{+}$уравнение $(56)$, в котором вектор-столбец правой части равен

$$
\mathbf{V}=\left(\xi / \omega \varepsilon_{3} ; \eta / \omega \varepsilon_{3} ; 0 ; 0\right)^{T} \text {. }
$$

Решая уравнение $(56)$, найдем $\hat{E}_{1, x}^{-}, \hat{E}_{1, y}^{-}$и $\hat{E}_{3, x}^{+}, \hat{E}_{3, y}^{+}$, и далее по формулам (49)-(54) при $N=2, z_{d}=z_{2}=d$ получим волну в дальней зоне вертикального диполя, расположенного на границе пленки, в призме (в область 1) и свободном полупространстве (область 3 ) над пленкой.

\section{Результаты численных расчетов для элементарных горизонтального и вертикального диполей, расположенных на границе пленки золота в схеме Кречмана}

Настоящая работа является продолжением работы по изучению явлений, связанных с поверхностной плазмонной волной, которая возбуждается по схеме Кречмана и распространяется вдоль свободной поверхности пленки металла (например, золота) определенной оптимальной толщины, нанесенной на призму [25]. На свободной поверхности пленки возбуждается волна, которая имеет высокую интенсивность на границе раздела и экспоненциально спадает в направлении, нормальном к границе. Если поместить на свободной поверхности молекулу или частицу, в которой будет индуцироваться поверхностной волной дипольный момент, то можно найти излучение индуцированного диполя, разложив диполь на параллельную и перпендикулярную границе составляющие.

Точное решение задачи излучения элементарного диполя, ориентированного параллельно и перпендикулярно границе плоскослоистой структуры, было исследовано в работах $[15,16]$. Интерес представляет излучение в 

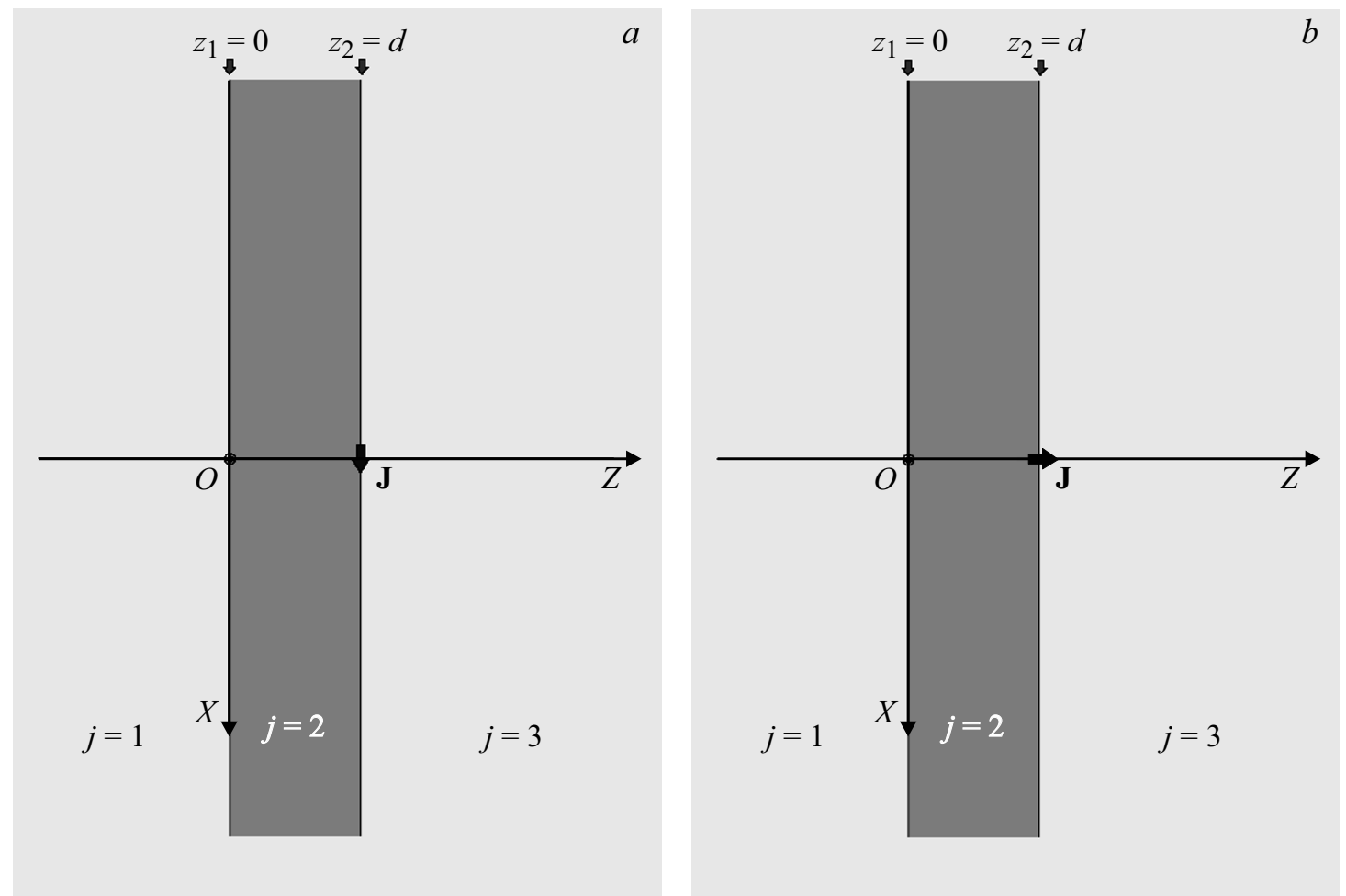

Рис. 6. Геометрия задачи излучения элементарного источника тока, расположенного на свободной границе пленки в схеме Кречмана, при ориентации дипольного момента параллельно $(a)$ и перпендикулярно границе $(b)$.

дальней зоне, именно оно будет попадать в микроскоп при исследовании излучения молекул или частиц на поверхности в схеме Кречмана. Однако вычисления полей в дальней зоне методом работ $[15,16]$ все же представляют определенные трудности, так как требуют интегрирования. Метод настоящей работы с вычислительной точки зрения значительно упрощает решение задачи нахождения излучения в дальней зоне и не требует интегрирования. Кроме того, этот метод без труда обобщается на задачи с любым числом пленок в слоистой структуре, а также на задачи, в которых диполь находится на любой границе любой из пленок, внутри любой из пленок или в окружающих полупространствах на конечном расстоянии от границы. В последнем случае нужно добавить в расчеты дополнительно пленку с параметрами полупространства и толщиной, равной расстоянию от источника до ближайшей границы структуры. Наконец, метод данной работы применим и для магнитных пленок, так как параметры пленок влияют только на матричные элементы соответствующих слоев.

Рассмотрим пространственную направленность излучения элементарных диполей, расположенных на свободной границе одной пленки, нанесенной на призму в схеме Кречмана. Рассмотрим два случая, первый диполи расположены на свободной границе пленки золота оптимальной толщины, по границе которой может распространяться поверхностная плазмонная волна, и второй - диполи расположены на границе двух по- лупространств без пленки (толщина пленки $d \rightarrow 0$ ). Излучение в дальней зоне будем характеризовать диаграммами направленности. Сравнение этих двух случаев поможет понять влияние поверхностной волны на направленные свойства элементарных излучателей.

Итак, выберем частоту излучения диполя $\omega$, соответствующую длине волны в вакууме, равной $\lambda_{0}=633 \mathrm{~nm}$, показатель преломления полупространства призмы на этой частоте выберем равным $n_{p}=1.6\left(\varepsilon_{1}=2.56 \varepsilon_{0}\right.$, где $\varepsilon_{0}$ - диэлектрическая проницаемость вакуума), показатель преломления свободного полупространства над пленкой $-n_{1}=1\left(\varepsilon_{3}=\varepsilon_{0}\right)$. Диэлектрическую проницаемость золотой пленки на заданной частоте примем равной $\varepsilon_{2}=(-11.6+i 1.2) \varepsilon_{0}[13]$, а ее оптимальную толщину $-d=48.6 \mathrm{~nm}$. Как было показано [25], при таких параметрах на границе пленки наилучшим образом возбуждается поверхностная плазмонная волна при падении плоской волны $p$-поляризации со стороны призмы под углом примерно $\alpha_{\text {opt }}=41.0^{\circ}$ к нормали. Для того чтобы оценить влияние такой пленки на излучение диполя в полупространство призмы и свободное полупространство, были вычислены диаграммы направленности горизонтального и вертикального диполей. Диаграммы вычислялись по амплитуде поля. Для горизонтального диполя диаграммы направленности находились в $E$ - и $H$-плоскостях, т.е. в плоскостях, нормальных границе, проходящих через центр диполя и проходящих вдоль и перпендикулярно направлению диполя соответственно. 

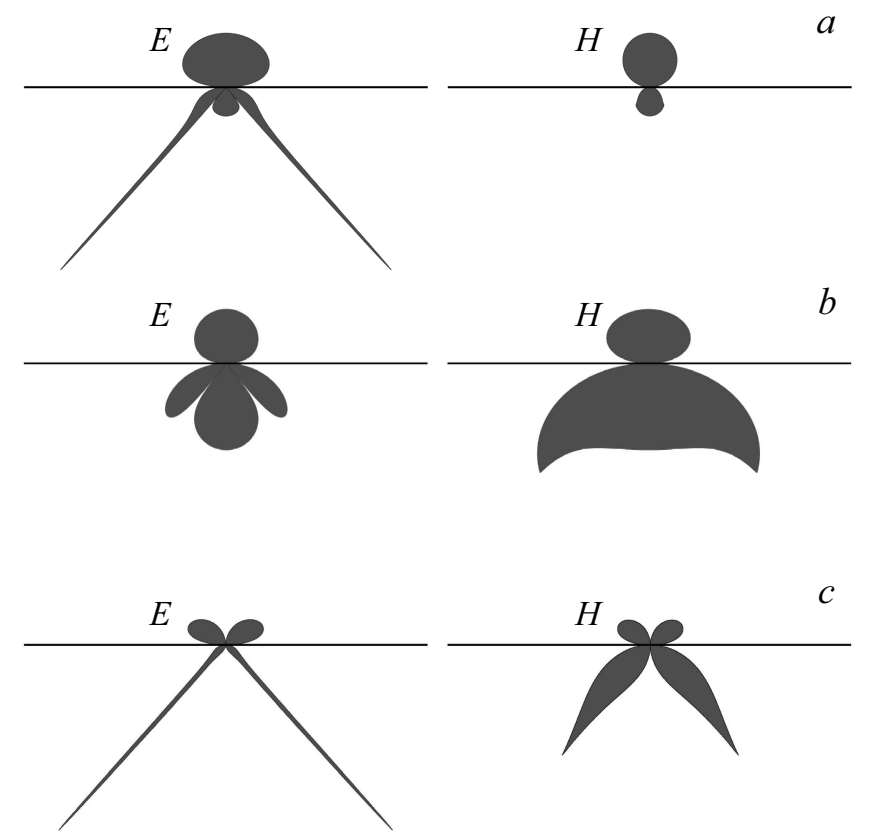

Рис. 7. Диаграммы направленности по полю в $E$ - и $H$ плоскостях, вычисленные для элементарного горизонтального диполя, расположенного на границе золотой пленки оптимальной толщины, нанесенной на призму $(a)$, на границе двух полупространств $(b)$, а также для вертикального диполя на золотой пленке (слева) и без пленки на границе двух полупространств (справа, $c)$.

На рис. 7, a показаны диаграммы направленности элементарного горизонтального диполя в полярных координатах. Диполь расположен на свободной границе золотой пленки с $\varepsilon_{2}$ со стороны полупространства с $\varepsilon_{3}$. Сверху от горизонтальной линии, которая представляет плоскость пленки, расположено свободное полупространство с $\varepsilon_{2}$, снизу - полупространство призмы с $\varepsilon_{1}$. Слева (рис. 7, a) представлена диаграмма направленности в $E$-плоскости, справа - в $H$-плоскости.

Для сравнения (на рис. 7,b) показаны диаграммы направленности элементарного горизонтального диполя на границе этих полупространств с $\varepsilon_{1}$ и $\varepsilon_{3}$, т.е. без пленки.

Из сравнения рис. 7, $a$ и 7, $b$ видно, что наличие металлической пленки и возбуждение поверхностной волны на ее поверхности (поверхностная волна имеет $p$-поляризацию и порождает излучение в призму под углом примерно 41) приводит к возникновению в диаграмме направленности в $E$-плоскости острых лепестков в сторону призмы под указанным углом. Наличие этих острых интенсивных лепестков говорит о существенном влиянии поверхностной волны на направленные свойства излучения диполя в $E$-плоскости. В $H$-плоскости поверхностная волна не возбуждается, и таких острых лепестков не возникает.

На рис. 7, с показана диаграмма направленности элементарного вертикального диполя, расположенного на свободной границе золотой пленки - на границе областей с проницаемостями $\varepsilon_{2}$ и $\varepsilon_{3}$ со стороны полупространства с $\varepsilon_{3}$ (слева) и расположенного на границе двух полупространств с диэлектрическими проницаемостями $\varepsilon_{1}$ и $\varepsilon_{3}$ со стороны полупространства с $\varepsilon_{3}$ (справа). Излучение вертикального диполя имеет осевую симметрию, и диаграммы направленности в любой плоскости, перпендикулярной границе и проходящей через диполь, одинаковы. Волны, исходящие от вертикального диполя, имеют $p$-поляризацию, и видно, что наличие металлической пленки и возбуждение поверхностной волны существенно влияют на диаграмму направленности в сторону призмы. Возникает узкий интенсивный лепесток диаграммы направленности по направляющей конуса с половинным углом при вершине примерно $41^{\circ}$.

Наконец, интегрированием по полусферам большого радиуса интенсивности уходящих волн было найдено отношение мощностей излучения диполей в свободное полупространство и в полупространство призмы. Для горизонтального и вертикального диполей на золотой пленке это отношение мощностей оказалось равным 0.909 и 0.548 соответственно. Для горизонтального и вертикального диполей на границе полупространств отношение мощностей получилось равным 0.163 и 0.149 соответственно. Полученные отношения мощностей показывают, что при наличии золотой пленки (в случае возбуждения поверхностной волны) заметная часть мощности уходит в свободное пространство в виде распространяющейся на бесконечность волны.

\section{Заключение}

Итак, предложено теоретическое рассмотрение задачи нахождения излучения в дальней зоне элементарного дипольного источника, расположенного на границе или внутри плоскослоистой среды. Показана общность и вычислительная эффективность метода. Рассмотрена прикладная задача - нахождение направленных характеристик излучения нанообъектов, находящихся на свободной поверхности пленки золота оптимальной толщины в схеме Кречмана. Показано, что наличие металлической пленки, в которой может возбуждаться поверхностная волна, существенно меняет диаграмму направленности излучателя в полупространство призмы, возникают интенсивные узконаправленные лепестки диаграммы направленности, связанные с возбуждающейся поверхностной волной.

\section{Список литературы}

[1] Kretschmann E., Raether H.Z. // Naturforsch. A. 1968. V. 23. P. 2135 .

[2] Raether H. Surface Plasmons. Berlin: Springer-Verlag, 1988. $353 \mathrm{p}$.

[3] Barnes W.L. // J. Opt. A: Pure Appl. Opt. 2006. V. 8. P. S87. 
[4] Garabedian R., Gonzalez C., Richards J. et al. // Sensors and Actuators A. 1994. V. 43. P. 202.

[5] Петрин А.Б. // Опт. и спектр. 2018. Т. 125. № 6. С. 830.

[6] Де Бройль Л. По тропам науки. М.: Изд. ин. литературы, 1962.

[7] Sommerfeld A. // Ann. Physik. (Leipzig). 1926. V. 81. P. 1135.

[8] Wait J.R. // IEEE Antennas and Propagation Magazine. 1998. V. 40. N 5. P. 7.

[9] King R.W.P., Smith G.S. Antennas in Matter. Cambridge, MA: M.I.T. Press, 1981.

[10] Wu T.T. // J. Appl. Phys. 1957. V. 28. N 3. P. 299.

[11] Jansen R.H. // IEEE Trans. Microwave Theory Tech. 1985. V. 33. N 10. P. 1043.

[12] King R.W.P. // IEEE Trans. Microwave Theory Tech. 1988. V. 36. N 6. P. 1080.

[13] Новотный Л., Хехт Б. Основы нанооптики. М.: Физматлит, 2009.

[14] Pendry J.B. // Phys. Rev. Let. 2000. V. 85. N 18. P. 3966.

[15] Петрин А.Б. // Опт. и спектр. 2020. Т. 128. № 11. С. 1676.

[16] Петрин А.Б. // ЖЭТФ. 2020. (принята к публикации.)

[17] Петрин А.Б. // ЖЭТФ. 2008. Т. 134. № 3(9). С. 436.

[18] Петрин А.Б. // Опт. и спектр. 2018. Т. 125. № 3. С. 375.

[19] Петрин А.Б. // Опт. и спектр. 2019. Т. 126. № 3. С. 350.

[20] Петрин А.Б. // Опт. и спектр. 2019. Т. 127. № 4. С. 654.

[21] Стрэттон Джс.А. Теория электромагнетизма. МоскваЛенинград, 1948.

[22] Stratton J.A., Chu L.J. // Phys. Rev. 1939. V. 56. P. 99.

[23] Schelkunoff S.A. // Phys. Rev. 1939. V. 56. P. 308.

[24] Harrington R.F. Time-Harmonic Electromagnetlc Fields. New York: McGraw-Hill, 1961.

[25] Петрин А.Б. // Опт. и спектр. 2019. Т. 127. № 6. С. 1051. 